\title{
Adaptive Optics and Optical Vortices
}

\author{
S. G. Garanin, F. A. Starikov and Yu. I. Malakhov \\ Additional information is available at the end of the chapter \\ http://dx.doi.org/10.5772/53328
}

\section{Introduction}

The achievement of minimal angular divergence of a laser beam is one of the most important problems in laser physics since many laser applications demand extreme concentration of radiation. Under the beam formation in the laser oscillator or amplifier with optically inhomogeneous gain medium and optical elements, the divergence usually exceeds the diffraction limit, and the phase surface of the laser beam differs from the plane surface. However, even if one succeeds in realizing the close-to-plane radiation wavefront at the laser output, the laser radiation experiences increasing phase disturbances under the propagation of the beam in an environment with optical inhomogeneities (atmosphere). These disturbances appear with the wavefront receiving smooth, regular distortions, the transverse intensity distribution becomes inhomogeneous, and the beam broadens out.

The correction of the laser radiation phase, which is a smooth continuous spatial function, can be performed using a conventional adaptive optical system including a wavefront sensor and a wavefront corrector. The wavefront sensor performs the measurement (in other words, reconstruction) of the radiation phase surface; then, on the basis of these data, the wavefront corrector (for example, a reflecting mirror with deformable surface) transforms the phase front in the proper way. If all components of the adaptive optical system are involved in the common circuit with the feedback, then the adaptive system is known as a closed-loop system. The adaptive correction of the wavefront with smooth distortions has a somewhat long history and considerable advances $[1,2,3,4,5,6]$.

When a laser beam passes a sufficiently long distance in a turbulent atmosphere, the socalled regime of strong scintillations (intensity fluctuations) is realized. Under such conditions the optical field becomes speckled, lines appear in the space along the beam axis where the intensity vanishes and the surrounding zones of the wavefront attain a helicoidal (screw) shape. If the intensity in an acnode of the transverse plane is zero, then the phase in 
this point is not defined. In view of its screw form, the phase surface in the vicinity of such point has a break, the height of which is divisible by the wavelength. Since the phase is defined accurate to the addend that is aliquot to $2 \pi$, it is formally continuous but under a complete circling on the phase surface around the singular point one cannot reach the starting place. The integration of the phase gradient over some closed contour encircling such singular point results in a circulation not equal to zero, in contrast to the null circulation at the usual smoothed-inhomogeneous regular phase distribution. The indicated properties represent evidence of strong distortions of the wavefront - screw dislocations or optical vortices. The vortical character of the beam is detected with ease in the experiment after the analysis of the picture of its interference with the obliquely incident plane wave: the interference fringes arise or vanish in the centers of screw dislocations forming peculiar "forks".

Scintillations in the atmosphere especially decrease the efficiency of light energy transportation and distort the information carried by a laser beam in issues of astronomy and optical communications. Scintillation effects present special difficulty for adaptive optics, and their correction is one of key trends in the development of state-of-the-art adaptive optical systems.

However it should be noted that the possibility to control the optical vortices (including the means of adaptive optics) presents interest not only for atmospheric optics but for a new optical field, namely, singular optics [7, 8, 9]. The fact is that optical vortices have very promising applications in optical data processing, micro-manipulation, coronagraphy, etc. where any type of management of the singular phase could be required.

This chapter is dedicated to wavefront reconstruction and adaptive phase correction of a vortex laser beam, which is generated in the form of the Laguerre-Gaussian $L G_{0}{ }^{1}$ laser mode. The content of the chapter is as follows. In Section 1 we specify the origin and main properties of optical vortices as well as some their practical applications. Section 2 is dedicated to a short description of the origination of optical vortices in a turbulent atmosphere and correspondent problems of the adaptive optics. In Section 3 some means are given concerning the generation of optical vortices under laboratory conditions, aimed at the formation of a "reference" optical vortex with the maximally predetermined phase surface, and the experimental results of such formation are illustrated. Section 4 is concerned with vortex beam phase surface registration, based on measurements of phase local tilts using a Hartmann-Shack wavefront sensor and a novel reconstruction technique. In Section 5 experimental results of correction of a vortex beam are demonstrated in the conventional closed-loop adaptive optical system including a Hartmann-Shack wavefront sensor and a bimorph deformable adaptive mirror. Conclusions summarize the abovementioned research results.

\section{Origin, main properties and practical applications of optical vortices}

The singularity of the radiation field phase $S$ is identified by the term "optical vortex", which can appear in the complex function $\exp \{i S\}$ representing a monochromatic light wave [10]. The amplitude of scalar wave field $A$ (and, correspondingly, its intensity $I=|A|^{2}$ ) in the point of the vortex location approaches zero. The phase of radiation $S$ changes its value by 
$2 \pi m$ with the encircling the singularity point clockwise or counter-clockwise, where $m$ is the positive or negative integer number known as the vortex "topological charge". In the centre of the vortex (intensity zero point) the phase remains indefinite. Such an optical singularity is the result of the interference of partial components of the wave field with a phase shift, which is initial or acquired during propagation in an inhomogeneous medium. In 3D space the points with zero intensity form zero lines. On these lines the potentiality of phase field is violated; and the regions of "defective" (singular) phase can be considered as vortex strings like the regions with concentrated vorticity, which are considered in the hydrodynamics of ideal liquid. We are interested in a case where the lines of zero intensity have a predominantly longitudinal direction, i.e. form a longitudinal optical vortex. The equiphase surface in the vicinity of such a line has the appearance of a screw-like (helicoidal) structure, threaded on this line. In the interference pattern of the vortex wave under consideration with any regular wave, the vortices are revealed through the appearance of so-called "forks" (i.e., branching of interference fringes), coinciding with zero points of the intensity.

Investigations of waves with screw wavefront and methods of their generation were reported as early as by Bryngdahl [11]. The theory of waves carrying phase singularities was developed in detail by Nye and Berry $[12,10]$, prompting a series of publications dealing with the problem (see $[13,14,7,8]$ and the lists of references therein). The term "optical vortex" was introduced in [15]. Along with the term "optical vortex", the phenomenon is also referred to as "wavefront screw dislocation". The latter appeared because of similarities between distorted wavefront and the crystal lattice with defects. The following terms are also used: "topological defects", "phase singularities", "phase cuts", and "branch cuts".

Thus the indication of the existence of an optical vortex in an optical field is the presence of an isolated point $\{\mathbf{r}, z\}$ in a plane, perpendicular to the light propagation axis, in which intensity $I(\mathbf{r}, z)$ is approaching zero, phase $S(\mathbf{r}, z)$ is indefinite, and integration of the phase gradient $\nabla_{\perp} S$ field over some closed contour $\Gamma$ encircling this point results in a circulation not equal to zero:

$$
\oint_{\Gamma} \nabla_{\perp} S(\boldsymbol{\rho}, z) d \boldsymbol{\rho}=2 \pi m
$$

where $d \varrho$ is the element of the contour $\Gamma$.

The propagation of slowly-varying complex amplitude of the scalar wave field $A$ in the free space is described by the well-known quasi-optical equation of the parabolic type (see, for example, [16]):

$$
\frac{\partial A}{\partial z}-\frac{i}{2 k} \frac{\partial^{2} A}{\partial r^{2}}=0
$$

where $k=2 \pi / \lambda$ is wave number, $\lambda$ is the radiation wavelength, $z$ is the longitudinal coordinate corresponding with the beam propagation axis, $\mathbf{r}=\mathbf{e}_{x} x+\mathbf{e}_{\mathrm{y}} y$ is the transverse radius-vec- 
tor. In the process of radiation propagation in the medium vortices appear, travel in space, and disappear (are annihilated).

Laguerre-Gaussian laser beams $L G_{n}{ }^{m}$ are related to the familiar class of vortex beams and are used most often in experiments with optical vortices [7-9]. They are the eigen-modes of the homogeneous quasi-optical parabolic equation (2) [17], so they do not change their form under the free space propagation and lens transformations. The correspondent solution of equation (2) in cylindrical coordinates $(r, \varphi, z)$ has the following form:

$$
A(r, \varphi ; z)=A_{0} \frac{w_{0}}{w}\left(\frac{r}{w}\right)^{m} \Phi_{m}(\varphi) L_{n}^{m}\left(2 \frac{r^{2}}{w^{2}}\right) \exp \left(-\frac{r^{2}}{w^{2}}+i \frac{z r^{2}}{z_{0} w^{2}}-i(2 n+m+1) \operatorname{arctg} \frac{z}{z_{0}}\right),
$$

The typical transverse size of the beam $w$ in (3) is determined by the relation $w^{2}=w_{0}^{2}\left[1+z^{2} /\right.$ $\left.z_{0}^{2}\right]$ where, in its turn, $w_{0}$ is the transverse beam size in the waist and $z_{0}=k w_{0}^{2} / 2$ is the typical waist length. The radial part of distribution (3) includes the generalized Laguerre polynomial

$$
L_{n}^{m}(x)=\frac{e^{x}}{x^{m} n !} \frac{d^{n}}{d x^{n}}\left(x^{n+m} e^{-x}\right)
$$

In addition to an item responsible for wavefront curvature and transversely-uniform Gouy phase, the angular factor $\Phi_{m}(\varphi)$ contributes to the phase part of distribution (3). The angular part of formula (3) is represented in the form of a linear combination of harmonic functions

$$
\Phi_{m}(\varphi)=c_{1} \cdot \Omega_{m}(\varphi)+c_{2} \cdot \Omega_{-m}(\varphi),
$$

where $\Omega_{m}(\varphi)=\exp (\operatorname{im} \varphi), \varphi=\operatorname{arctg}(y / x)$ is the azimuth angle in the transverse plane. The $c_{1}$ and $c_{2}$ constants determine the beam character and the presence of singularity in it.

Let's consider two cases of the angular function $\Phi_{m}(\varphi)$ distribution from (5) at $m>0$. In the first case, when $c_{1}=1$ and $c_{2}=0$, we have $\Phi_{m}(\varphi)=\exp (\operatorname{im} \varphi)$. The form of helicoidal phase surface assigned by the $m \varphi$ function (we do not take into account the wavefront curvature in (3) determining the beam broadening or narrowing only) in the vortex Laguerre-Gaussian laser beam is presented in Figure 1. This phase does not depend on $r$ at the given $\varphi$ and rises linearly with $\varphi$ increasing. In the phase surface the spatial break of $m \lambda$ (or $2 \pi m$ radians) depth is present. Under the complete circular trip around the optical axis on the phase surface it is impossible to get to the starting point. As it has been commented above, such a shape of the phase factor is what causes the singular, vortex behavior of the beam. The positive or negative sign of $m$ determines right or left curling of the phase helix.

On the optical axis, in the vortex center, the intensity is zero, resulting from the behavior of the radial dependence of (3) and generalized Laguerre polynomial (4). The beam intensity distribution in the transverse plane, as it is seen from (3), is axially-symmetrical (modulus of $A$ depends on $r$ only) and visually represents the system of concentric rings. In the simplest 
case at $n=0, m=1$ ( $L G_{0}{ }^{1}$ mode) the intensity distribution has a doughnut-like form that is shown in Figure 2. Figure 2 also presents a picture of the interference of the given vortex laser beam with the obliquely incident monochromatic plane wave. In the picture, fringe branching is observed in the beam center with the "fork" formation (fringe birth) typical for screw dislocation [7-9]. At the arbitrary $m$ number the quantity of fringes that are born corresponds with this number, the double, triple, and other "forks" are formed. The presence of "forks" in the interference patterns of such kind is the standard evidence of the vortex nature of the beam in the experiment.

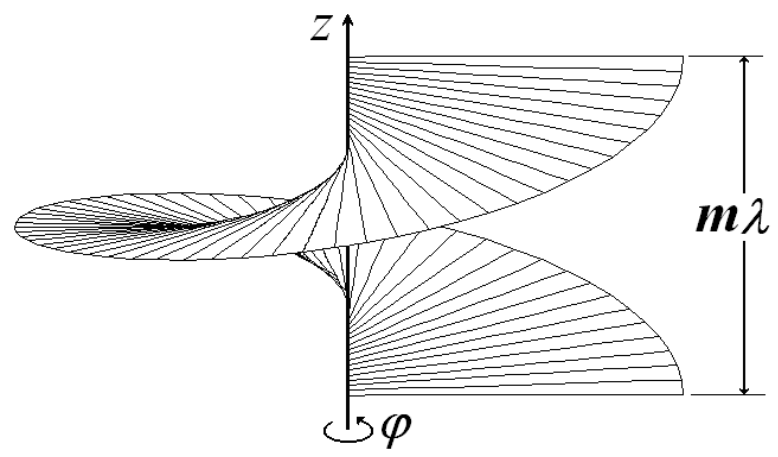

Figure 1. Phase surface shape of a Laguerre-Gaussian beam carrying an optical vortex.
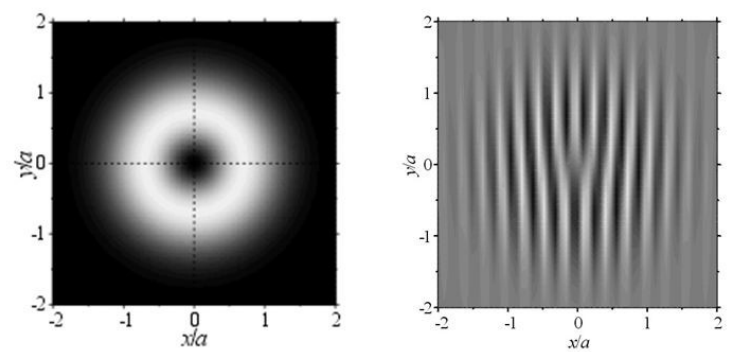

Figure 2. The intensity distribution in the Laguerre-Gaussian laser beam $L G_{n}{ }^{m}$ and the picture of its interference with an obliquely incident plane wave at $n=0, m=1, \Phi_{m}=\exp (i \varphi)$. 

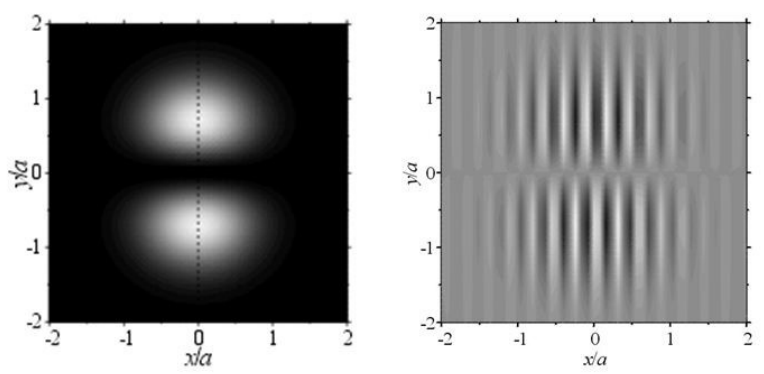

Figure 3. The intensity distribution in the Laguerre-Gaussian laser beam $L G_{n}{ }^{m}$ and the picture of its interference with an obliquely incident plane wave at $n=0, m=1, \Phi_{m}=\sin (i \varphi)$.

Let's consider one more case when $c_{1}=-c_{2}=1 / 2 i$ in (5), then $\Phi_{m}(\varphi)=\sin (m \varphi)$. Under such conditions, according to (3) the intensity distribution has no axial symmetry depending on $\varphi$. In Figure 3 the beam intensity distribution in the transverse plane at $\Phi_{m}=\sin \varphi$ for $n=0, m=1$ is shown. The phase portrait of the beam is also given in Figure 3. The half-period shift of interference fringes in the lower half plane as compared to the upper one demonstrates the phase asymmetry with respect to the $x$ axis. The fringe numbers in the lower and upper half planes are the same, i.e. the fringe birth does not take place. It is seen that the edge rather than screw phase dislocation occurs here since the phase distribution is step-like with a break of $\pi$ (instead of $2 \pi$ !) radians. The given example of the Laguerre-Gaussian beam demonstrates that at $\Phi_{m}=\sin \varphi$ the beam is not the vortical in nature. There is no singular point in the beam transverse section but there is a particular line $(y=0)$ where the intensity is zero. At $m>1$ in the beam there are $m$ such lines passing through the optical axis and dividing the transverse beam section by $2 m$ equal sectors. The radiation phase in each sector is uniform and differs in $\pi$ in the neighboring sectors.

Light beams with optical vortices currently attract considerable attention. This attention is encouraged by the extraordinary properties of such beams and by the important manifestations of these properties in many applications of science and technology.

It is known for a long time that light with circular polarization possesses an orbital moment. For the single photon its quantity equals $\pm \hbar$, where $\hbar$ is the Planck constant. However only relatively recently it was shown $[18,19]$ that light can have an orbital moment irrespective of its polarization state if its azimuth phase dependence is of the form $S=m \varphi$ where $\varphi$ is azimuth coordinate in the transverse cross section of the beam and $m$ is the positive or negative integer. The authors [18] supposed that the moment of each photon is defined by the formula $L=m \hbar$. As this phase dependence is the characteristic feature of the helical wavefront (the form of which is presented in Figure 1) so the beam carrying the optical vortex and possessing such phase front has to own the non-zero orbital moment $m \hbar$ per photon. The quantity of $m$ is defined by the topological charge of optical vortex.

The concept of orbital moment is not new. It is well known that multipole quantum jumps can results in the emission of radiation with orbital moment. However, such processes are 
infrequent and correspond to some forbidden atomic and molecular transitions. However, generating the beam carrying the optical vortex, one can readily obtain the light radiation beam with quantum orbital moment. Such beams can be used in investigations of all kinds of polarized light. For example, the photon analogy of spin-orbital interaction of electrons can be studied and in general it is possible to organize the search for new optical interactions. As the $m$-factor can acquire arbitrary values, any part of the beam (even one photon) can carry an unlimited amount of information coded in the topological charge. Thus the density of information in a channel where coding is realized with the use of orbital moment could be as high as compared with a channel with coding of the spin states of a photon. Because only two circular polarization states of the photon are possible, one photon can transmit only one bit of information. Presently, optical vortices have generated a great deal of interest in optical data processing technologies, namely, the coding/decoding in optical communication links in free space [20,21], optical data processing [22], optical interconnects [23], and quantum optics information processing [24].

The next practical application of optical vortices is optical micromanipulations and construction of so called optical traps, i.e. areas where the small (a few micrometers) particles can be locked in [25, 26]. Progress in the development of such traps allows the capture of particles of low and large refraction indexes [27]. Presently, this direction of research finds further continuation $[28,29,30]$.

It is also possible to use optical vortices to register objects with small luminosity located near a bright companion. Shadowing the bright object by a singular phase screen results in the formation of a window, in which the dim object is seen. The optical vortex filtration of such a kind was proposed in [31]. Using this method the companion located at 0.19 arcsec near the object was theoretically differentiated with intensity of radiation $2 \times 10^{5}$ times greater [32]. The possibility to use this method to detect planets orbiting bright stars was also illustrated by astronomers [33,34]. Vortex coronagraphy is now undergoing further development [35, 36]. There are a number of examples of non-astronomical applications [37, 38].

It was proposed to use optical vortices to improve optical measurements and increase the fidelity of optical testing [39, 40], for investigations in high-resolution fluorescence microscopy [41], optical lithography [42, 43], quantum entanglement [44, 45, 46], Bose-Einstein condensates [47].

Optical vortices show interesting properties in nonlinear optics [48]. For example, in [49, 50, 51] it was predicted that the phase conjugation at SBS of vortex beams is impossible due to the failure of selection of the conjugated mode. For a rather wide class of the vortex laser beams a novel and interesting phenomenon takes place which can be called the phase transformation at SBS. In essence there is only one Stokes mode, the amplification coefficient of which is maximal and higher than that of the conjugated mode. In other words, the non-conjugated mode is selected of in the Stokes beam. The principal Gaussian mode, which is orthogonal to the laser vortex mode, is an example of such an exceptional Stokes mode. The cause of this phenomenon is in the specific radial and azimuth distribution of the vortex laser beam. It is interesting that the hypersound vortices are formed in the SBS medium in ac- 
cordance with the law of topologic charge conservation. The predicted effects have been completely confirmed experimentally $[52,53,54]$.

\section{Optical vortices in turbulent atmosphere and the problem of adaptive correction}

In early investigations [12] it was shown that the presence of optical vortices is a distinctive property of the so called speckled fields, which form when the laser beam propagates in the scattering media. Experimental evidence of the existence of screw dislocations in the laser beam, passed through a random phase plate, were obtained in $[55,56,57]$ where topological limitations were also noted of adaptive control of the laser beams propagating in inhomogeneous media.

Turbulent atmosphere can be represented as the consequence of random phase screens. Under propagation in the turbulent atmosphere the regular optical field acquires rising aberrations. These aberrations manifest themselves in the broadening and random wandering of laser beams; the intensity distribution becomes non-regular and the wavefront deviates from initially set surface. These deformations of the wavefront can be corrected using adaptive optics. To this end, effective sensors and correctors of wavefront were designed [1-6]. The problem becomes more complicated when the laser beam passes a relatively long distance in a weak turbulent medium or if the turbulence becomes too strong. In this case optical vortices develop in the beam; the shape of the wavefront changes qualitatively and singularities appear.

The influence of the scintillation effects are determined (see, for example, $[2,4]$ ) by the closeness to unity of the Rytov variance

$$
\sigma_{\chi}^{2} \approx 0.56 k^{7 / 6} \int_{0}^{L} C_{n}^{2}(z) z^{5 / 6}(z / L)^{5 / 6} d z,
$$

where $C_{n}^{2}(z)$ describes the dependence of structure constant of the refractive index fluctuations over the propagation path and $L$ is the path length. The regime of strong scintillations is not realized when $\sigma_{x}{ }^{2}<<1$.

Figure 4 demonstrates the results of numerical simulation of propagation of a Gaussian laser beam $(\lambda=1 \mu)$ in turbulent atmosphere in a model case of invariable structure constant $C_{n}^{2}$ $=10^{-14} \mathrm{~cm}^{-2 / 3}$ for the distance of $1 \mathrm{~km}$ when the regime of strong scintillations is realized according to (6). The steady-state equation for the slowly-varying complex field amplitude $A$ differs from the equation (2) by the presence of the inhomogeneous term: 


$$
\frac{\partial A}{\partial z}-\frac{i}{2 k} \frac{\partial^{2} A}{\partial r^{2}}+\frac{i k}{2}(\tilde{\varepsilon}-1) A=0
$$

where $\tilde{\varepsilon}$ is the fluctuating dielectric permittivity of the turbulent atmosphere. In numerical simulations we use the finite-difference algorithm of numerical solving of parabolic equation (7) described in [58]. It is characterized by an accuracy, which considerably exceeds the accuracy of the widespread spectral methods [59]. The amplitude error of an elementary harmonic solution of the homogeneous equation is equal to zero, whereas the phase error is significantly reduced and proportional to the transverse integration step to the power of six. To take into account the inhomogeneous term of the equation, the splitting by physical processes is employed. The effect of randomly inhomogeneous distribution of dielectric permittivity is allowed for using a model of random phase screens, which is commonly used in calculations of radiation propagation in optically inhomogeneous stochastic media [60]. The spatial spectrum of dielectric permittivity fluctuations is described taking into account the Tatarsky and von Karman modifications of the Kolmogorov model [61].

The fragment of speckled distribution of optical field intensity after the propagation is shown in Figure 4. Dark spots are seen where the intensity vanishes. As it has been noted before, the presence of optical vortices in the beam is easily detected, based on the picture of its interference with an obliquely incident plane wave. The correspondent picture is shown in Figure 4 as well. In the centers of screw dislocations the fringe branching is observed, i.e. the birth or disappearance of the fringes takes place with formation of typical "forks" in the interferogram (compare with Figure 2). There are also zones of edge dislocations (compare with Figure 3). The number, allocation and helicity of the vortices in the beam are random in nature but the vortices are born as well as annihilated in pairs. If the initial beam is regular (vortex-free), then the total topological charge of the vortices in the beam will be equal to zero in each transverse section of the beam along the propagation path in accordance with the conservation law of topological charge (or orbital angular moment) [7-9].
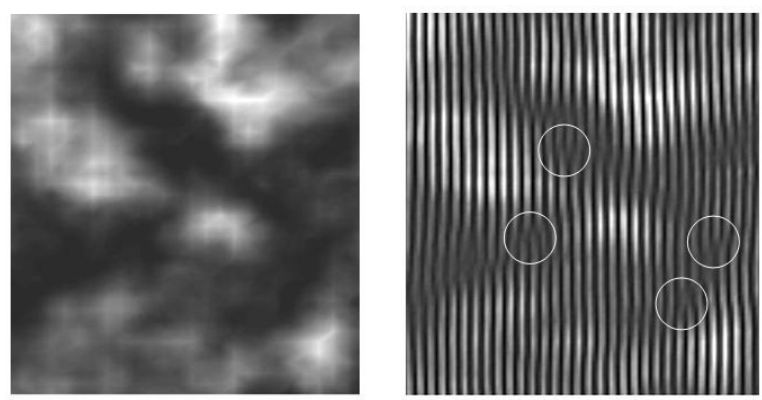

Figure 4. Optical vortices in the laser beam after atmospheric propagation: the speckled intensity distribution and the picture of interference of the beam with the obliquely incident plane wave including "forks" denoted by light circles. 
One of the first papers dealing with the appearance of optical vortices in laser beams propagating in randomly inhomogeneous medium was published by Fried and Vaughn in 1992 [62]. They pointed out that the presence of dislocations makes registration of the wavefront more difficult and they considered methods for solving the problem. In 1995 the authors of Ref. [63] encountered this problem in experimental investigations of laser beam propagation in the atmosphere. It was shown that the existence of light vortices is an obstacle for atmospheric adaptive optical systems. After that it was theoretically shown that screw dislocations give rise to errors in the procedure of wavefront registration by the Shack-Hartmann sensor $[64,65]$. Due to zero amplitude of the signal in singular points, the information carried by the beam becomes less reliable and the compensation for turbulent aberrations is less effective [66]. Along with [63], the experimental investigation [67] can be taken here as an example where the results of adaptive correction are presented for distortions of beams propagating in the atmosphere.

Since one of the key elements of an adaptive optical system is the wavefront sensor of laser radiation, there is a pressing need to create sensors that are capable of ensuring the required spatial resolution and maximal accuracy of the measurements. In this connection there is necessity need to develop algorithms for measurement of wavefront with screw dislocations, which are sufficiently precise, efficient and economical given the computing resources, and resistant to measurement noises. The traditional methods of wave front measurements [1-6] in the event of the above-mentioned conditions are in fact of no help. The wavefront sensors have been not able to restore the phase under the conditions of strong scintillations [68]. The experimental determination of the location of phase discontinuities itself already generates serious difficulties [69]. In spite of the fact that the construction features of algorithms of wavefront recovery in the presence of screw dislocations were set forth in a number of theoretical papers $[68,69,70,71,72,73,74,75]$, there were not many published experimental works in this direction. Thus, phase distribution has been investigated in different diffraction orders for a laser beam passed through a specially synthesized hologram, designed for generating higher-order Laguerre-Gaussian modes [76]. An interferometer with high spatial resolution was used to measure transverse phase distribution and localization of phase singularities. The interferometric wavefront sensor was applied also in a high-speed adaptive optical system to compensate phase distortions under conditions of strong scintillations of the coherent radiation in the turbulent atmosphere [77] as well as when modelling the turbulent path under laboratory conditions [78]. In [77, 78] the local phase was measured, without reconstructing the global wavefront that is much less sensitive to the presence of phase residues. The interferometric methods of phase determination are rather complicated and require that several interferograms are obtained at various phase shifts between a plane reference wave and a signal wave. It is noteworthy, however, that in the adaptive optical systems [1-6] the HartmannShack wavefront sensor $[79,80]$ has a wider application compared with the interferometric sensors including the lateral shearing interferometers [81, 82], the curvature sensor [83, 84, 85], and the pyramidal sensor $[86,87]$. The cause of this is just in a simpler and more reliable arrangement and construction of the Hartmann-Shack sensor. However, there have been practically no publications of the results of experimental investigations connected with applications of this sensor for measurements of singular phase distributions. 
The problem of a wavefront corrector (adaptive mirror) suitable for controlling a singular phase surface is also topical. In the adaptive optical systems [77, 78] the wavefront correctors were based on the micro-electromechanical system (MEMS) spatial light modulators with the large number of actuators. The results of $[77,78]$ shown that continuous MEMS mirrors with high dynamic response bandwidth, combined with the interferometric wavefront sensor, can ensure a noticeable correction of scintillation. However, the MEMS mirrors are characterized by low laser damage resistance that can considerably limit applications. The bimorph or pusher-type piezoceramics-based flexible mirrors with the modal response functions of control elements have a much higher laser damage threshold [3-5]. Recently [88] a complicated cascaded imaging adaptive optical system with a number of bimorph piezoceramic mirrors was used to mitigate turbulence effect basing, in particular, on conventional Hartmann-Shack wavefront sensor data. Conventional adaptive compensation was obtained in [88] which proved to be very poor at deep turbulence. The scintillation and vortices may be one of the causes of this.

In the investigations, the results of which are described in this chapter, the development of an algorithm of the Hartmann-Shack reconstruction of vortex wavefront of the laser beam plays a substantial role. The creation of efficient algorithms for the wavefront sensor of vortex beams implies the experiments under modeling conditions when the optical vortices are artificially generated by special laboratory means. Moreover, as long as the matter concerns the creation of a new algorithm of wavefront reconstruction, it is possible to estimate its accuracy only under operation with the beam, the singular phase structure of which is known in detail beforehand. The formation of optical beams with the given configuration of phase singularities and their transformations is one of main trends in the novel advanced optical branch - singular optics [7-9].

Thus, the first stage of the research sees the generation of a vortex laser beam with the given topological charge. In our case the role of this beam is played by the single optical vortex, namely, the Laguerre-Gaussian mode. Further, at the second stage, with the help of the Hartmann-Shack wavefront sensor, the task of registration of the vortex beam phase surface is solved using the new algorithm of singular wavefront reconstruction. Finally, at the third stage, the correction of the singular wavefront is undertaken in a closed-loop adaptive optical system, including the Hartmann-Shack wavefront sensor and the wavefront corrector in the form of a piezoelectric-based bimorph mirror.

\section{Generation of optical vortex}

As it has been indicated above, to examine the accuracy of the wavefront reconstruction algorithm and its efficiency in the experiment itself a "reference" vortex beam has to be formed with a predetermined phase surface. This is important as, otherwise, it would be impossible to make sure that the algorithm recovers the true phase surface under conditions when robust alternative methods of its reconstruction are missing or unavailable. The Laguerre-Gaussian vortex modes $L G_{n}{ }^{m}$ can play the role of such "reference" optical vortices. 
To create a beam with phase singularities artificially from an initial plane or Gaussian wave, a number of experimental techniques have been elaborated. There are many papers concerning the various aspects of generation of beams with phase singularities (see, for example, $[89,90,91,92,93,94,95,96,97,98,99,100,101,102,103,104])$. Among other possibilities, we can also refer to several methods for phase singularity creation in the optical beams based on nonlinear effects $[105,106,107,108]$. The generation of optical vortices is also possible in the waveguides $[109,110,111]$. The adaptive mirrors themselves can be used for the formation of optical vortices [112, 113]. In this chapter, though, we dwell only on a number of ways to generate the vortex beams, which allow one to form close-to-"reference" vortices with well-determined singular phase structure that is necessary for the accuracy analysis of the new algorithm of Hartmann-Shack wavefront reconstruction.

One method for generation of the screw dislocations is by forming the vortex beam immediately inside a laser cavity. The authors of [114] were the first to report that the generation of wavefront vortices is possible using a cw laser source. It was shown in [115] that insertion of a non-axisymmetric transparency into the cavity results in generation of a vortex beam. It was reported in [116] that a pure spiral mode can be obtained by introducing a spiral phase element (SPE) into the laser cavity, which selects the chosen mode. The geometry of the cavity intended for generation of such laser beams from [116] is shown in Figure 5. Here a rear mirror is replaced by a reflecting spiral phase element, which adds the phase change +2 im $\varphi$ after reflecting. As a result of reflection, the phase of a spiral mode $-\operatorname{im} \varphi$ changes to $+i m \varphi$. The cylindrical lens inside the cavity is focused on the output coupler. This lens inverts the helicity of the mode back to the field described by $\exp \{-i m \varphi\}$ and ensures the generation of the required spiral beam. The beam at the output passes another cylindrical lens and its distribution becomes the same as inside the cavity. A pinhole in the cavity ensures the generation of a spiral mode of minimal order, i.e., $T E M_{01}$ mode. It should be stressed that the spiral phase element determines the parameters of the spiral beam within the cavity so that by its variation the parameters of the output beam can be controlled.

This method was tested with a linearly polarized $\mathrm{CO}_{2}$-laser. The reflecting spiral phase element was made of silicon by multilevel etching. It had 32 levels with the entire height of break $\lambda$ that corresponds to $m=1$. Precision of etching was about $3 \%$ and deviation of the surface from the prescribed form was less than $20 \mathrm{~nm}$. The reflecting coefficient of the element was greater than $98 \%$; the diameter and length of the laser tube were $11 \mathrm{~mm}$ and $65 \mathrm{~cm}$, respectively. The lens inside the cavity with focal length $12.5 \mathrm{~cm}$ was focused in the output concave mirror with a radius of $3 \mathrm{~m}$. An identical lens was placed outside the cavity to collimate the beam. In Figure 6 we demonstrate the stable spiral beam obtained in the experiment [116]. The vortical nature of the beam is proved not by demonstration of the "fork" in the interferogram but by the doughnut-like intensity distribution in the near and the far zone. 


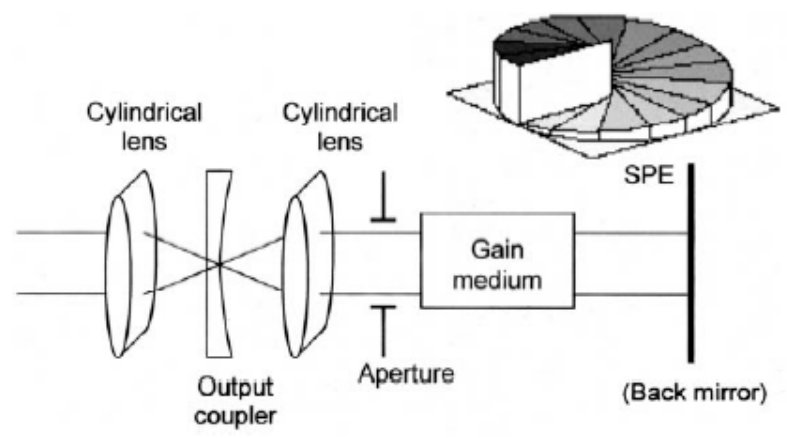

Figure 5. Configuration of a laser cavity intended for generation of spiral beams [116].
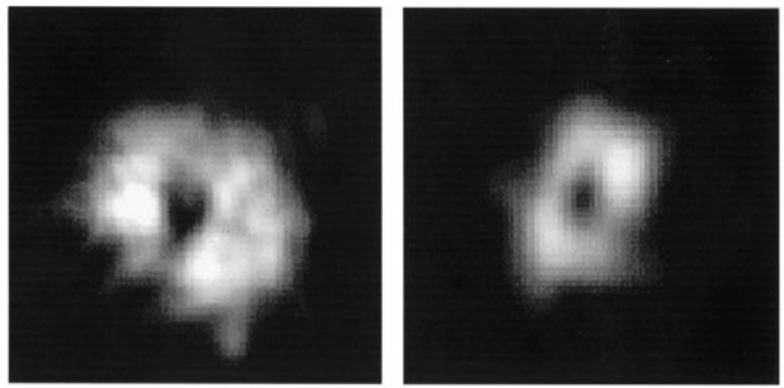

Figure 6. Intensity distribution of vortex beam generated in the laser cavity in near (left) and far (right) zone [116].

A ring cavity with the Dove's prism can also be used to generate vortex beams. It was shown [117] that modes of such a resonator are singular beams.

The next way to generate the optical vortex uses a phase (or a mode) converter. Usually it transforms a Hermit-Gaussian mode, generated in the laser, into a corresponding LaguerreGaussian mode. This method was first proposed in [118]. In the experiment the authors used a cylindrical lens, the axis of which was placed at an angle of $45^{\mathrm{o}}$ with respect to the $H G_{01}$ mode for conversion into a $L G_{0}{ }^{1}$ mode. The incident mode appears to the lens as a superposition of $H G_{01}$ mode parallel to the lens axis and a mode perpendicular to the axis. The mode perpendicular to the lens axis passes through a focus, advancing the relative Gouy phase between the two modes of $\pi / 2$ as required to form the doughnut mode from uncharged $H G_{01}$ and $H G_{10}$ modes.

In Ref. [91] an expression was derived for an integral transformation of Hermit-Gaussian modes into Laguerre-Gaussian modes in the astigmatic optical system, and it was shown theoretically that passing the beam through the cylindrical lens can perform the conversion. The theory of a $\pi / 2$ mode converter and a $\pi$ mode converter, produced by two cylindrical 
lenses, was described in more details in [92]. Padgett et al. describe in a tutorial paper [119] how a range of Laguerre-Gaussian modes can be produced using two cylindrical lenses starting from the corresponding Hermit-Gaussian modes, and present the clear examples, showing the intensity and phase distributions obtained. The initial higher order HermitGaussian modes can be produced in the laser with intracavity cross-wires. The authors of [96] used a similar technique with a Nd:YAG laser operating at the $100 \mathrm{~mW}$ level.

Even in the absence of the required initial $H G_{10}$ mode in the laser emission, it is easy to produce artificially a similar configuration by introducing a glass plate in a half of the $T E M_{00}$ beam and achieving the necessary $\pi$ phase shift, and then to apply mode conversion. An example of such doughnut beam creation was reported in [120]. The efficiency of conversion was about $50 \%$. It is possible to use the cylindrical lens mode converter [121] but with production of the initial higher order Hermit-Gaussian mode, by exciting it in an actively stabilized ring cavity, matching in the Gaussian beam from their titanium sapphire laser. The efficiency was up to $40 \%$ for the $L G_{0}{ }^{1}$ mode, and higher order doughnuts could also be obtained easily. The method proposed in [122] is based on the formation of a pseudo $H G_{N M}$ mode, propagating the Gaussian beam of a number of edges of thin glass plates and forming the edge dislocations with the following its astigmatic conversion into a Laguerre-Gaussian mode.

It was reported in [123] that in the event of ideal conversion, the efficiency of Hermit-Gaussian mode transformation into Laguerre-Gaussian mode is about $99.9 \%$. The spherical aberration does not reduce the efficiency factor. Typically cylindrical lenses are not perfect and their defects give rise to several Laguerre-Gaussian modes. The superposition of components can be unstable and this means a dependence of intensity on the longitudinal coordinate. If special means are not employed the precision of lens fabrication is about $5 \%$, in this case the efficiency of beam transformation into Laguerre-Gaussian mode is $95 \%$. Imperfections of $10 \%$ result in drop of efficiency down to $80 \%$.

In $[124,54]$ the formation of the Laguerre-Gaussian $L G_{0}{ }^{1}$ or $L G_{1}{ }^{1}$ modes was performed at the output of a pulsed laser-generator of Hermit-Gaussian $H G_{01}$ or $H G_{21}$ modes with the help of a tunable astigmatic $\pi / 2$-converter based on the so-called optical quadrupole [125]. It consists of two similar mechano-optical modules, each of which incorporates the positive and negative cylindrical lenses with the same focal length and a positive spherical lens. The mechanical configuration of each module can synchronously turn the incorporated cylindrical lenses in the opposite directions with respect to the optical axis, which ensures its rearrangement. In the initial position the optical forces of cylindrical lenses completely compensate each other and their axes coincide with the main axes of the intensity distribution of the laser. The distance between the modules is fixed so that the spherical lenses in different modules are located at a focal distance from each other and form the optical Fourier transformer.

To study the phase structure of radiation, in [54] use was made of a special interferometer scheme, where the reference beam was produced from a part of the original Laguerre-Gaussian $L G_{0}{ }^{1}$ or $L G_{1}{ }^{1}$ mode (see Figure 7). As a result, each of the modes interfered with a similar one, but with a topological charge of the opposite sign (the opposite helicity), i.e., with $L G_{0}^{-1}$ or $L G_{1}^{-1}$ mode. The interference fringe density depended on the thickness of the plane-parallel plate 1 and could be additionally varied by inclining the mirrors 2 (see figure 7). 
The peculiarity of the interference of two Laguerre-Gaussian modes, having the opposite helicity of the phase, manifests itself in the branching of a fringe in the middle of the beam and formation of a characteristic "fork" with an additional fringe appearing in the centre, as compared with the case of a vortex mode interfering with a plane reference wave (see Figure 2). Such branching of fringes indicates the vortex nature of the investigated beam, while the absence of branching is a manifestation of the regular character of the beam phase surface.

Figure 8 displays the experimental distributions of intensity of the laser mode $L G_{0}{ }^{1}$ in far field and its picture of interference with an obliquely incident wave in the form of $L G_{0}^{-1}$ mode.

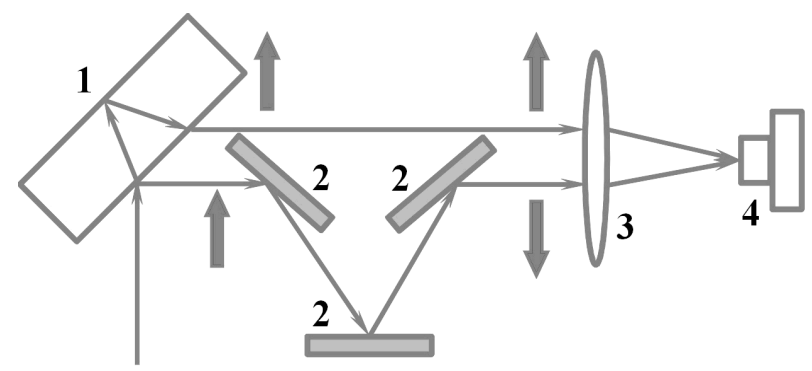

Figure 7. The optical scheme for registration of the phase portrait of a laser beam [54]: 1 - dividing parallel-sided plate, 2 - mirrors, 3 - lens, 4 - CCD camera.
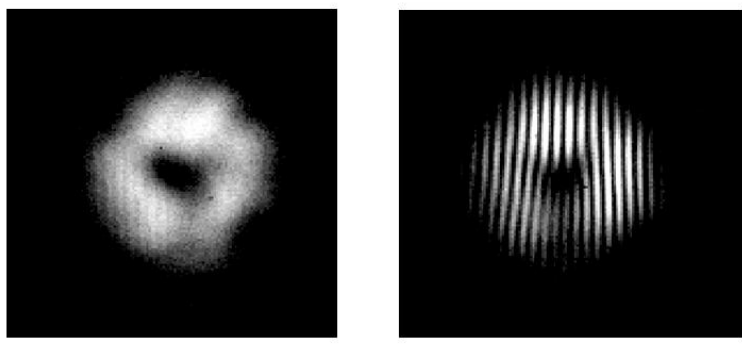

Figure 8. The experimental distribution of intensity and phase portrait of the laser mode $L G_{0}{ }^{1}$ obtained at usage of a phase converter [54].

The invention of a branched hologram $[89,93]$ uncovered a relatively easy way to produce beams with optical vortices from an ordinary wave by using its diffraction on the amplitude diffraction grating. The idea of singular beam formation is based on the holographic principle: a readout beam restores the wave, which has participated in the hologram recording. Instead of writing a hologram with two actual optical waves, it is sufficient to calculate the interference pattern numerically and, for example, print the picture in black-and-white or grey scale. The amplitude grating after transverse scaling can, when illuminated by a regular wave, reproduce singular beams in diffraction orders. 
Using the description of the singular wave amplitude (2), one can easily calculate the pattern of interference of such wave with a coherent plane wave tilted by the angle $\gamma$ with respect to the $z$ axis. The calculated interference pattern depends on the angle $\gamma$ between the interfering waves and corresponds to two well-known holographic schemes: on-axis [126] when $\gamma=0$ and off-axis [127] holograms. The spiral hologram (or spiral zone plate) realized under the on-axis scheme suffers from all the disadvantages inherent to the on-axis holograms, namely, the lack of spatial separation of the reconstructed beams from the directly transmitted readout beam. Therefore the on-axis spiral holograms have not found wide application unlike the off-axis computer-generated holograms [128].

Under interference between the plane wave and the optical vortex with unity topologic charge the transmittance of amplitude diffraction grating varies according to

$$
T=\left[\frac{1}{2}\left[1-\cos \left(\left(\frac{2 \pi x}{\Lambda}\right)-\operatorname{arctg}\left(\frac{y}{x}\right)\right)\right]\right]^{2}
$$

where $\Lambda=\lambda / \gamma$ is the grating period. When the basic Gaussian mode passes through the grating and is focused by the lens, the $L G_{0}{ }^{1}$ and $L G_{0}{ }^{-1}$ modes occur in the far field in the $1^{\text {st }}$ and $1^{\text {st }}$ orders of diffraction, respectively. The period of the grating should be equal to $100-200$ microns to separate the orders of diffraction properly in the actual experiment.

The two simplest ways to fabricate the amplitude diffraction gratings in the form of computer-synthesized holograms are as follows. The first involves the printing of an image onto a transparency utilized in laserjet printers. The second approach consists in photographing an inverted image, printed on a sheet of white paper, onto photo-film. Fragments of images of the gratings with the profile (8) obtained upon usage of the laser transparency with the resolution of 1200 ppi as well as the photo-film are shown in Figure 9 [129, 130, 131]. The usage of the photo-film is more preferable since it gives higher quality of the vortex to be formed and greater power conversion coefficient into the required diffraction order.

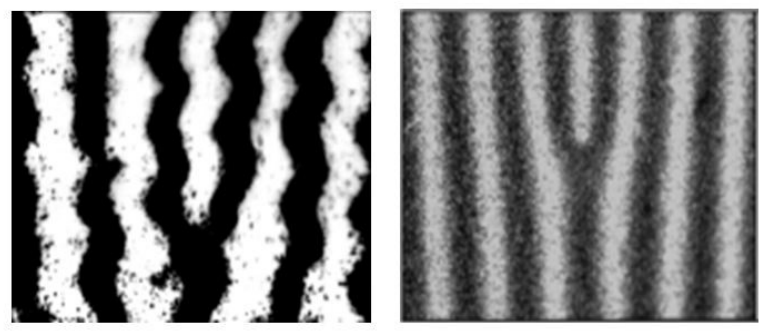

Figure 9. Magnified fragment of the amplitude grating in the experiment with laser transparency (left) and photofilm (right). 


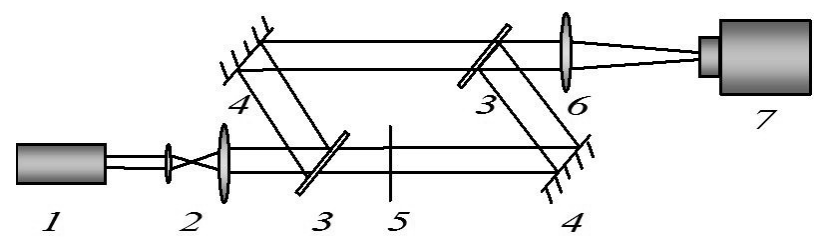

Figure 10. The set-up scheme for formation of the optical vortex:1 - He-Ne laser; 2 - collimator; 3 - optical plane plate, 4 - reflecting plane mirror; 5 - amplitude grating forming the optical vortex; 6 - lens; 7 - CCD camera.

The experimental set-up scheme for formation of the optical vortex with the help of computer-synthesized amplitude grating is shown in Figure 10. The experimental set-up consists of a system for forming the collimated laser beam $(\lambda=0.633 \mu)$, a Mach-Zehnder interferometer and a registration system of the far field beam intensity and interference pattern. The system for forming the collimated beam includes the He-Ne laser 1 and a collimator 2 consisting of two lenses forming the Gaussian beam with a plane wave front. The Mach-Zehnder interferometer consists of two plane plates 3 and two mirrors 4 . The computer-synthesized amplitude diffraction grating 5 is inserted into one of the arms of the interferometer. The lens 6 focuses the radiation onto the screen of CCD-camera 7. When blocking or admitting the reference beam from the second arm of the interferometer, the CCD camera registers the far field intensity of the vortex beam or its interference pattern with the reference beam, respectively. Varying the angle between both these beams allows one to vary the interference fringe density.

After passing the beam through the optical scheme, the central peak (0-th diffraction order) is formed in the far-field zone. It concerns the non-scattered component of the beam that has passed through the grating. Less intensive two doughnut-shaped lateral peaks are formed symmetrically from the central peak. The lateral peaks represent the optical vortices and have a topological charge equal to the value and opposite to the sign. In the $1^{\text {st }}$ order of diffraction there is only $16.7 \%$ of energy penetrating the grating in an ideal scenario. In the experiment this part of energy is equal to about $10 \%$ owing to the imperfect structure of the grating and its incomplete transmittance.

For registration of the optical vortex it is necessary to cut off the unnecessary diffraction orders. The pictures of doughnut-like intensity distribution of the optical vortex (the lateral peak) in far field and its interference pattern are shown in Figure 11. The rigorous proof of that the obtained lateral peaks bear the optical vortices is the availability of typical "fork" in the interference pattern. The formed vortex in Figure 11, as the vortices in Figures 6 and 8, is rather different from the ideal $L G_{0}{ }^{1}$ mode that is seen from their comparison with Figure 2. 

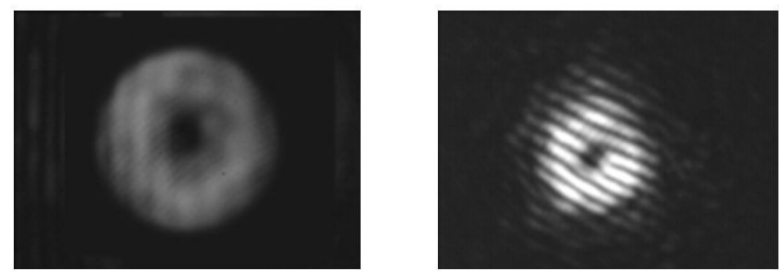

Figure 11. The intensity of the lateral diffraction order in the far field and interference pattern with the plane wave in the case of computer-synthesized amplitude diffraction grating.

Phase transparencies can be used to generate optical vortices. Application of the phase modulator results in phase changes and, after that, in amplitude changes with deep intensity modulation and the advent of zeros. In [132] the optical schematic was described, in which the wave carrying the optical vortex is recorded on thick film (Bregg's hologram) that is used to reproduce the vortex beam. The diffraction efficiency in this schematic is about $99 \%$. A relatively thin transparency with thickness varied gradually in one of the half planes is used in the other method [133]. The efficiency of this method is greater than $90 \%$. A similar method was proposed in [134], but a dielectric wedge was used as the phase modulator. In general, a chain of several vortices is formed as the product of this process. The shape deformation of each vortex depends on the wedge angle and on the diameter of the beam waist on the wedge surface. Varying the waist radius, one can obtain the required number of vortices (even a single vortex).

One more method of the optical vortex generation was proposed in [95]. In this method a phase transparency is used, which immediately adds the artificial vortex component into the phase profile. One such phase modulator is a transparent plate, one surface of which has a helical profile, repeating the singular phase distribution. To obtain Laguerre-Gaussian mode the depth of break onto the surface should be equal to $m \lambda /\left(n_{1}-n_{2}\right)$, where $n_{1}$ is the plate index of refraction, and $n_{2}$ is the medium index of refraction. If these conditions are met, the optical vortex appears in the far field. The main difficulty of this method lies in the problems of fabricating such a transparency. A special mask is used in the manufacture of such plates, which is made negative relative to the spiral phase plate to be formed [135]. The mask is made of brass and checked by the control interferometric system of high precision. After fabrication the mask is filled with a polymer substance and covered by glass. The spiral phase plate is formed on the glass as a result of polymerization. The transfer coefficient of such a plate is 0.98 . Publications have appeared recently concerning the generation of phase transparencies using liquid crystals [136, 137].

We note that the manufacture of phase modulators is a special branch of optics called kinoform optics. At the heart of this branch lays the possibility to realize the phase control of radiation by a step-like change of the thickness or the refraction index of some structures [138]. Light weight, small size, and low cost are the most attractive features of kinoform phase elements, when compared with lenses, prisms, mirrors, and other optical devices. The kinoforms can be described as optical elements performing phase modulation with a depth 
not greater than the wavelength of light. This aim is realized by jumps of the optical path length not less than the even number of half wavelengths. These jumps form the lines dividing the kinoform into several zones. In boundaries of each zone the optical path length can be constant (there are two levels of binary phase elements), they can change discretely ( $n$ level phase elements), or they can change more continuously (in an ideal phase element $n$ approaches infinity). With an increase of $n$ the phase efficiency of the element increases as well as its ability to control light properly. The application of kinoforms facilitates a reduction in the number of optical elements in the system by combining optical properties of several elements into one kinoform. Thus, these optical methods offer broad potential for anyone who wants to obtain beams with desired properties and to generate beams with optical vortices.

The fabrication of spiral (or helicoidal) phase plates techniques has progressed in recent years $[139,140,141]$. We will describe the generation of a doughnut Laguerre-Gaussian $L G_{0}{ }^{1}$ mode with the help of a spiral phase plate [129, 130, 142, 143, 144] manufactured with etching of the fused quartz substrate using kinoform technology. Quartz displays a high damage threshold at $\lambda=0.3-1.3 \mu$, high uniformity of chemical composition and refractive index $n$ that minimizes the laser beam distortions on passing.

The fabrication of a kinoform spiral phase plate of fused quartz is performed as follows [142]. A quartz plate, $3 \mathrm{~cm}$ in diameter and $3 \mathrm{~mm}$ in thickness, is taken as the substrate. Both surfaces of the substrate are mechanically polished with a nanodiamond suspension up to the flatness better than $\lambda / 30$. Special precautions are made to avoid the formation of surface damage layer that may destabilize subsequent etching. A multi-level stepped microrelief, imitating the continuous helicoidal profile, is fabricated using precise sequential etchings of the surface through a photoresist mask in a mixture based on hydrofluoric acid. At every stage, a level pattern is formed in the photoresist layer using a method of deep UV photolithography. The temperature during etching is stabilized with an accuracy of $\pm 0.1^{\circ} \mathrm{C}$. The $16-$ and 32-level spiral phase plates at $m=1$ and 2 have been fabricated. As the calculations show, such stepped plates and an ideal plate with an exactly helicoidal surface give practically the same optical vortices in the far field. As contrasted to the examples of spiral phase plates [140], the plates [142] have a very high laser damage threshold and a working diameter of 2 $\mathrm{cm}$ that is larger by an order of magnitude. The high laser damage resistance of such plates allows their use in experiments with powerful laser beams [52-54]. The general view of the spiral phase plate is shown in Figure 12.

The 3D image of the central part of a 32-level spiral phase plate designed for $\lambda=0.633 \mu, m=1$ is shown in Figure 13. As it is seen from Figure 13, upon motion around the plate axis along a circle (perpendicular to the propeller bosses), the change of the etched profile altitude is linear with a rather high accuracy. The total break height of the microrelief on the plate surface of $1317.5 \mathrm{~nm}$ agrees with the calculated value $\lambda /(n-1)=1339.6 \mathrm{~nm}$, where $n$ is the substrate refraction index, with an accuracy about of one and a half percent. As the measurements show, the roughness of the etched and non-etched surfaces (including the deepest one) is approximately the same. The roughness rms of each step surface equals 1-1.5 $\mathrm{nm}$, amounting to $2-3 \%$ of the height of one step of $43.2 \mathrm{~nm}$. 


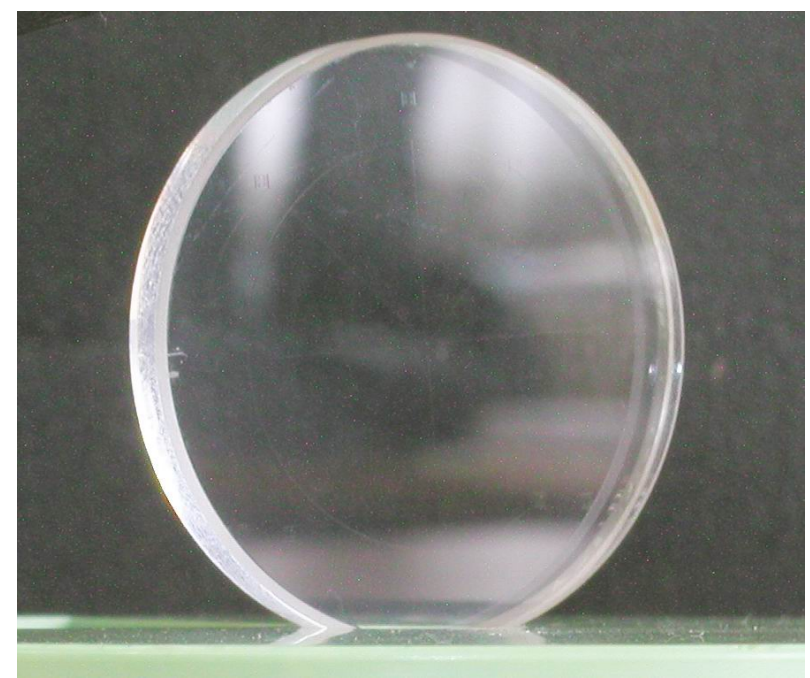

Figure 12. The photo-image of the 32-level spiral phase plate.
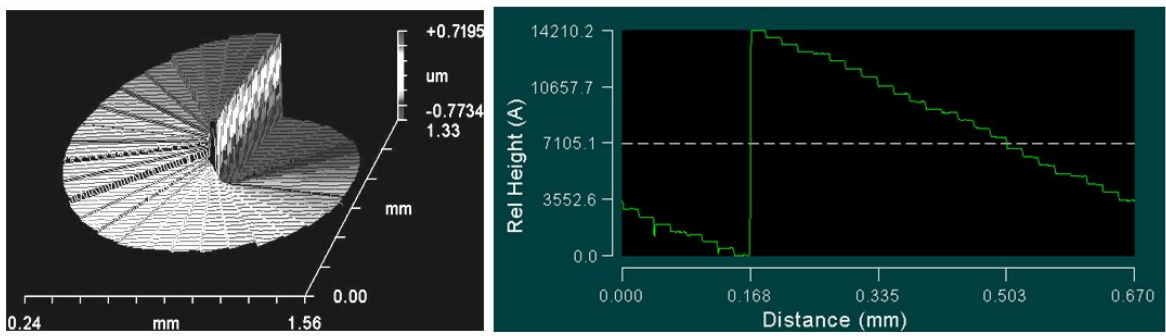

Figure 13. The image of surface in the near-axis region of the 32-level phase plate designed for $\lambda=0.633 \mu$ and its profile shape under motion along the circular line.

It should be noted that a laser beam in the form of a principal Gaussian mode with a plane wavefront that passes through a spiral phase plate maximally resembles the $L G_{0}{ }^{1}$ mode in the focal plane of a lens (in far field). The part of this mode in the beam exceeds more than $90 \%$; the residual energy is confined in general in the higher Laguerre-Gaussian modes $L G_{n}{ }^{1}$. It should be noted that the proper intensity modulation of the beam incident to the plate can additionally enhance the portion of the $L G_{0}{ }^{1}$ mode.

To generate a vortex beam with the help of a spiral phase plate, the experimental setup shown in Figure 10 is used. The spiral phase plate is installed into the scheme instead of the amplitude diffraction grating. In this case a vortex is formed in the $0^{\text {th }}$ diffraction order in far field. Figure 14 demonstrates the experimental distributions of laser intensity in the far field and the pattern of interference of this beam with a obliquely reference plane wave. It is seen 
that the beam intensity distribution has a true doughnut-like shape. The wavefront singularity appears, as before, by fringe branching in the beam center with the forming of a "fork" typical for screw dislocation with unity topological charge.
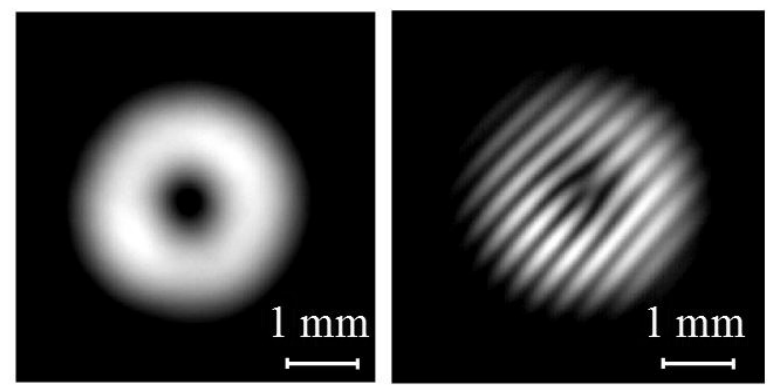

Figure 14. Experimental distribution of intensity of a vortex beam in far field and its interference pattern with obliquely incident reference plane wave in an experiment with a kinoform spiral phase plate.

The experimental data are in good agreement with the results of numerical simulation of the optical system, taking into account the stepped structure of spiral phase plate. The results barely differ from the distribution shown in Figure 2. It should be noted that the vortex quality (similarity to $L G_{0}{ }^{1}$ mode) is very good, caused by the high surface quality of the spiral phase plate throughout its area. This circumstance gives us grounds to believe that the vortex wave front to be reconstructed by the Hartmann-Shack sensor has to be close to the ideal $L G_{0}{ }^{1}$ wave front.

\section{Wavefront sensing of optical vortex}

The problem of phase reconstruction using the Shack-Hartmann technique was successfully solved for optical fields with smooth wavefronts [145, 146, 147]. In the simplest case, to obtain the phase $S(\mathbf{r})$ using the results of measurement of phase gradient projection $\nabla_{\perp} S_{m}(\mathbf{r})$ on the transverse plane it is possible to employ the numeric integration of the gradient over a contour $\Gamma$ :

$$
S(\boldsymbol{r})=S_{0}(\boldsymbol{r})+\int_{\Gamma} \nabla_{\perp} S_{m}(\rho) d \rho,
$$

where $\mathrm{r}=\{x, y\}$. Since for the ordinary wave fields the phase distribution is the potential function, the values of $S(\mathrm{r})$ do not depend formally on the configuration of the integration path. In the actual experiments, however, some errors are always present, so the potentiality of phase is violated and the results of phase reconstruction depend on the integration path [147]. To reduce the noise influence on the results it was proposed to consider the phase re- 
construction as the minimization of a certain functional. The most commonly used functional is the criterion corresponding to the minimum of the weighted square of residual error of gradient of the reconstructed phase $\nabla_{\perp} S(\mathbf{r})$ and phase gradients $\nabla_{\perp} S_{m}(\mathbf{r})$, obtained in the measurements:

$$
\int_{D}\left(W(r) \cdot\left(\nabla_{\perp} S(r)-\nabla_{\perp} S_{m}(r)\right)^{2} \mathrm{~d} r \rightarrow \min \right.
$$

where $\mathbf{W}(\mathbf{r})=\left\{W_{x}(x, y), W_{н}(x, y)\right\}$ is the vector weighting function introduced to account for the reliability of $\nabla_{\perp} S_{m}(\mathbf{r})$ measurements. This method is known as the least mean square phase reconstruction.

The approaches to the solution of variation problem (10) are well known [146, 147] and actually mean the solution of the Poisson equation written with partial derivatives. Allowing for the weighting function $\mathrm{W}(\mathbf{r})$, it acquires the following form:

$$
W_{x}(x, y)\left(\frac{\partial S}{\partial x}-\frac{\partial S_{m}}{\partial x}\right)+W_{y}(x, y)\left(\frac{\partial S}{\partial y}-\frac{\partial S_{m}}{\partial y}\right)=0
$$

where $\partial S / \partial x$ and $\partial S / \partial y$ are gradients of reconstructed phase, $\partial S_{m} / \partial x$ and $\partial S_{m} / \partial y$ are measured gradients of the phase.

There are a wide variety of methods $[145,146,147,148]$ which can be used to solve the discrete variants of equation (11). For example, one can use the representation of (11) as a system of algebraic equations, the fast Fourier transform, or the Gauss-Zeidel iteration method applied to the multi-grid algorithm. This group of methods is equally well adopted for the application of centroid coordinates measured by the Shack-Hartmann sensor as input data:

$$
\left\{\nabla_{\perp} S_{m}(\boldsymbol{r}, z)\right\}_{I}=\frac{\iint V\left(\boldsymbol{r}-\boldsymbol{r}_{0}\right) I\left(\boldsymbol{r}_{0}\right) \nabla_{\perp} S\left(\boldsymbol{r}_{0}\right) d \boldsymbol{r}_{0}}{\iint V\left(\boldsymbol{r}-\boldsymbol{r}_{0}\right) I\left(\boldsymbol{r}_{0}\right) d \boldsymbol{r}_{0}},
$$

where the integration is performed over the square of the subaperture, $V$ is a subaperture function, and $I\left(\mathbf{r}_{0}\right)$ is intensity of the input beam.

The sensing of wavefront with screw phase dislocations by the least mean square method is not agreeable. With this technique (along with other methods based on the assumption that phase surface is a continuous function of coordinates) it is possible to reconstruct only a fraction of the entire phase function. As it turned out [68, 149], the differential properties of the vector field of phase gradients help to find some similarity between this field and the field of potential flow of a liquid penetrated by vortex strings. It is also possible to represent this vector field as a sum of potential and solenoid components: 


$$
\nabla_{\perp} S(\mathbf{r})=\nabla_{\perp} S_{p}(\mathbf{r})+\nabla_{\perp} S_{c}(\mathbf{r})
$$

where $\nabla_{\perp} S_{p}$ is the gradient of potential phase component and $\nabla_{\perp} S_{c}$ is the gradient of vortex (solenoidal) component. By using only the ordinary methods of phase reconstruction it is possible to reproduce just the part of phase distribution that corresponds to potential component in (13).

However, if the quantity $\nabla_{\perp} S_{c}$ is considered as a rotor of vector potential $\mathbf{H}$, namely, $\nabla_{\perp}$ $S_{c}=\nabla \times \mathrm{H}$, which is dependent only on the coordinates of optical vortices [68] then potential phase component in (13) can be found by the least mean square method [68, 147]. By means of a novel "hydrodynamic" approach to the properties of the vector filed of phase gradients a new group of methods was formed [72, 150,151], employing the discovered coordinates of dislocations and reconstructing the potential phase component with the least mean square method. Within another technique [74, 152] reproduction of the scalar potential is also based on the least square method but the vortex component is calculated with Eq. (9) via a consistent rotor of vector potential. The method of matching the vortex component was proposed in [153] and is based on the following equation:

$$
\nabla^{2}\left(\operatorname{Rot}_{-\pi / 2}\left(\nabla_{\perp} S_{c}\right)\right)=(\nabla \times \mathbf{H}) \cdot \mathbf{e}_{z}
$$

where $\mathbf{e}_{z}$ is the unit vector of $z$ axis and $\operatorname{Rot}_{-\pi / 2}\left(\nabla_{\perp} S_{c}\right)$ is operation of the rotation of each vector on $-\pi / 2$ angle. This relation is a Poisson equation which allows one to find components of consistent vectors of vortex phase gradient. Now it is possible to take Eq. (9) and obtain a vortex phase component, assuming that the consistent gradients of vortex phase are measured without errors.

The searching for dislocation located positions, which is required in algorithms of phase reconstruction [72, 150,151], is a sufficiently difficult problem. Because of the infinite phase gradients in the points of zero intensity, the application of methods based on solution of (13) $[74,152]$ is also not straightforward. Presently there is no such an algorithm, which guarantees the required fidelity of wavefront reconstruction in the presence of dislocations [64]. However, according to some estimations [154, 155, 156] the accurate detection of vortex coordinates and their topological charges insures the sensing of wavefronts with high precision. Therefore we expect a future improvement in reconstruction algorithms by involving more sophisticated methods into the consideration of gradient fields, insuring more accurate detection of dislocation positions and their topological charges.

Analysis shows that from the point of view of experimental realization, of the considered approaches of wavefront reconstruction the algorithm of D. Fried [74] is one of the best algorithms (with respect to accuracy, effectiveness and resistance to measurement noises) of recovery of phase surface $S(x, y)$ from its measured gradient $\nabla S_{\perp}$ distribution in the presence of optical vortices. Fried's algorithm (a noise-variance-weighted complex exponential reconstructor) consists of three parts: reduction or simplification, solving, and reconstruction. The algo- 
rithm designed for work in Hadjin geometry reconstructs the phase in the nodes of a quadratic grid with the dimensions $\left(2^{N}+1\right) \times\left(2^{N}+1\right)$, using the phase differences between these nodes. Obviously, to employ the algorithm we need $\left(2^{N}+1\right) \times 2^{N}$ and $2^{N} \times\left(2^{N}+1\right)$ array of phase differences along $x$ and $y$ axes. The words "complex exponential" mean that the phase reconstruction problem is reformulated to a task of recovery of "phasors" $u$ (the complex number with a unity absolute value and an argument that is equal to the phase of optical field) distribution in transverse section of the beam. Here, the analysis and transformation of differential complex vectors (differential phasors) $\Delta_{x} u \equiv \exp \left(i \Delta_{x} \varphi\right), \Delta_{y} u \equiv \exp \left(i \Delta_{y} \varphi\right)$, corresponding to phase differences $\Delta_{x} \varphi, \Delta_{y} \varphi$ between different nodes of the computational grid, are used. The words "noise-variance-weighted" mean that the algorithm takes into account the distinctions of measurement variance of individual differential phasors, i.e. the influence ("weight") of differential phasors on the recovery result is inversely proportional to their variance. This feature of Fried's algorithm allows us to apply it to a computational grid of arbitrary dimension, not only to the $\left(2^{N}+1\right) \times\left(2^{N}+1\right)$ grid [74]; to take into account the average statistical inequality of measurement errors of phase gradient in different areas of the beam (for example, on the sub-apertures of the Hartmann-Shack sensor) if the repeated characterization of the same beam is performed; to consider a prior concept of the inequality of measurement errors of phase gradient in these areas if the measurement of the beam characteristics is single.

In Fried's algorithm the differential phasors are unit vectors. The operation of normalization of a complex vector is applied to provide for this requirement. However, the amplitudes of differential phasors and phasors, obtained under reduction and reconstruction, contain information about measurement errors of phase differences in the actual experiment. Based on this reason the algorithm in question has been modified [157, 158, 159]. The modification involves exclusion of the operation of complex vector normalization and allows an increase in algorithm accuracy.

The experimental setup for registration of an optical vortex wavefront consists of a system for formation of collimated laser beam, the Mach-Zehnder interferometer (as in the scheme in Figure 10), and the additionally induced the Hartmann-Shack wavefront sensor [160, 161]. It is shown in Figure 15. The system of formation of collimated beam includes a He-Ne laser 1 ( $\lambda=0.633$ мкм) and collimator 2 composed of lenses with focal lengths $5 \mathrm{~cm}$ and $160 \mathrm{~cm}$. The collimator forms the reference basic Gaussian beam with a diameter of $1 \mathrm{~cm}$ and the plane wave front. The Mach-Zehnder interferometer includes two optical plates 3 and two mirrors 4. The spiral phase plate 5 for formation of the optical vortex is interposed into one of the interferometer arms. The working surface of the phase plate, with a diameter of $2 \mathrm{~cm}$ completely covers the beam. After passing through the spiral phase plate the Gaussian beam turns into an optical vortex (the $L G_{0}{ }^{1}$ mode) in the focal plane 8 of the lens 6, i.e. in far field, with high conversion coefficient. Focal plane 8 of the lens 6 with focal length $700 \mathrm{~cm}$ is transferred (for purposes of magnification) by an objective 8 in the optically conjugated plane $8^{\prime}$. The wavefront sensor consists of a lenslet array 9 situated in the plane 8'and a CCD camera 10. 


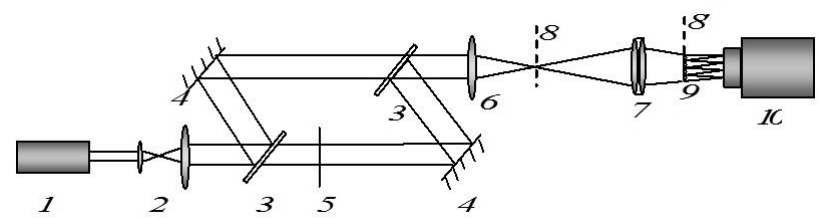

Figure 15. Experimental setup for wavefront sensing of optical vortex in far field: 1 - He-Ne laser; 2 - collimator; 3 optical plate; 4 - plane mirror; 5 - spiral phase plate; 6 - the lens $F=6$ m; 7 - the objective; 8 and $8^{\prime}$ - focal plane of lens 6 and its optically conjugated plane, respectively; 9 - lenslet array; 10 - CCD camera.

A technical feature of the Hartmann-Shack wavefront sensor used involves the employment of a raster of 8-level diffraction Fresnel lenses as the lenslet array (see Figure 16). The raster is fabricated from fused quartz by kinoform technology, similar to the aforesaid spiral phase plate, with the minimum size of microlens $d=0.1 \mathrm{~mm}$ and diffraction efficiency up to $90 \%$ [162]. The accuracy of etching profile depth is not worse than $2 \%$, the difference of the focal spot size from the theoretical size is $\sim 1 \%$. The spatial resolution of the wavefront sensor and its sensibility depend on the microlens geometry, the number of registered focal spots and their size with respect to the CCD camera pixel size.
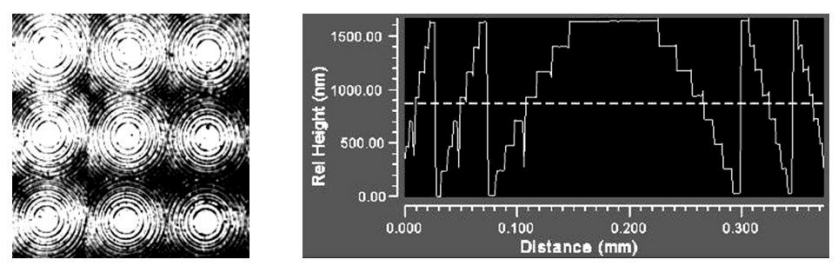

Figure 16. Photo image of a fragment of the lenslet array and image of surface profile of a microlens.

Under the registration of phase front the reference beam in the second arm of the interferometer is blocked. In the beginning the wavefront sensor is calibrated by a reference beam with plane phase front (the spiral phase plate is removed from the scheme). Then the spiral phase plate is inserted, and the picture of focal spots correspondent to singular phase front is registered. From the values of displacement of focal spots from initial positions, the local tilts of wave front on the sub-apertures of lenslet array are determined.

Experiments with a different number of registration spots on the hartmannogram have been carried out [160, 161]. When using a lenslet array with subaperture size $d=0.3 \mathrm{~mm}$, focal length $f=25 \mathrm{~mm}$ and $d=0.2 \mathrm{~mm}, f=15 \mathrm{~mm}$, the picture from $8 \times 8$ and $16 \times 16$ focal spots on the CCD camera screen has been registered, respectively. The results of experimental measurements of wave front gradients are given in Figure 17 for measurement points $8 \times 8$, where the picture of displacements of focal spots in the hartmannogram is shown. The vortex center is situated between the sub-apertures of the array. Displacement of each spot is demonstrated by the arrow (line segment). The arrow origin corresponds to the reference spot position 
whereas the arrow end corresponds to spot position after the insertion of a spiral phase plate in the experimental scheme. In Figure 17 the results are also shown, which are obtained in calculation and are correspondent to the ideal $L G_{0}{ }^{1}$ mode with high accuracy. It is seen from Figure 17 that the experimental and calculated pictures of spots' displacements agree with each other. Some local data difference is caused by the distinction between the phase and amplitude structure of the beam incident on the phase plate in the calculation and actual experiment, by the inaccurate location of the vortex in the optical axis assumed in calculations and by the inevitable noises of the measurement.

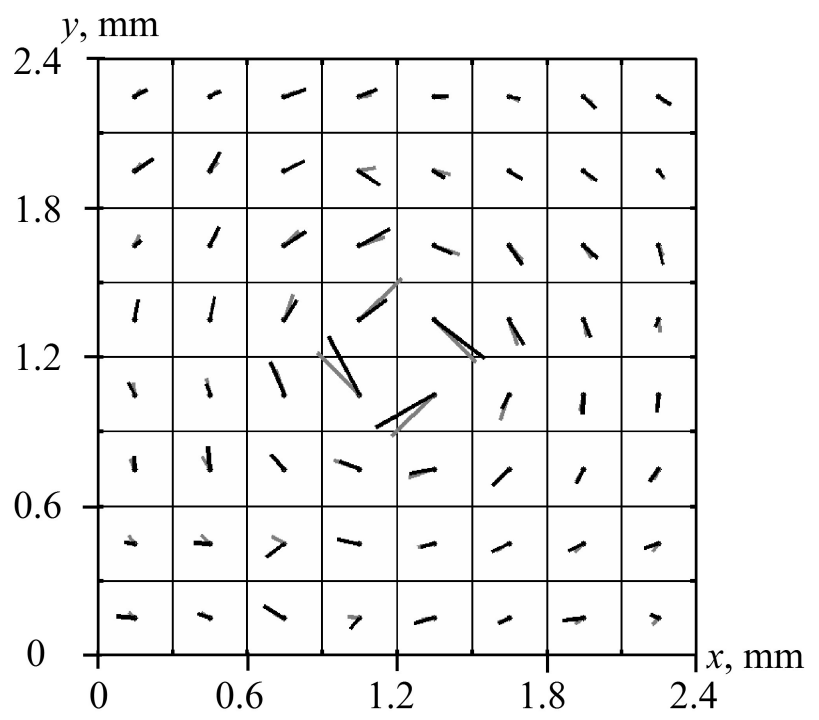

Figure 17. The picture of displacements of focal spots of the hartmannogram in experiment (black arrows) and calculation (grey arrows).

In Ref. [163] the vortex-like structure of displacements of spots in the hartmannogram was registered for the $L G_{0}{ }^{1}$ and higher-order modes. As the primary information, the spot displacements can be used for deriving the Poynting vector skews (in fact, wavefront tilts), as it was made in [163], as well as for wavefront sensing that is more nontrivial. In this chapter we simply consider the reconstruction of singular phase surface by the Hartmann-Shack sensor and describe the realization of this operation with the new reconstruction technique.

In Figure 18 we present the wave front surface of optical vortex reconstructed by the Hartmann-Shack sensor $[161,164]$ with software incorporating the code of restoration of singular phase surfaces [157-159]. Comparison of experimental data with calculated results shows that the wave front surface is restored by the actual Hartmann Shack wavefront sensor with good quality despite the rather small size of the matrix of wave front tilts (spots in the hartmannogram). The reconstructed wave front has the characteristic spiral form with a break of the surface about $2 \pi$. Analysis shows that the accuracy of wave front reconstruction (of 
course, from the viewpoint of its proximity to the theoretical results) is not worse than $\lambda / 20$. The accuracy of recovery of phase surface break increases at the measurement spots of 16x16. For comparison purposes in Figure 18 the result is demonstrated of the vortex wavefront reconstruction with the help of the standard least-squares restoration technique in the Hartmann-Shack sensor. It is seen that the conventional approach obviously fails.
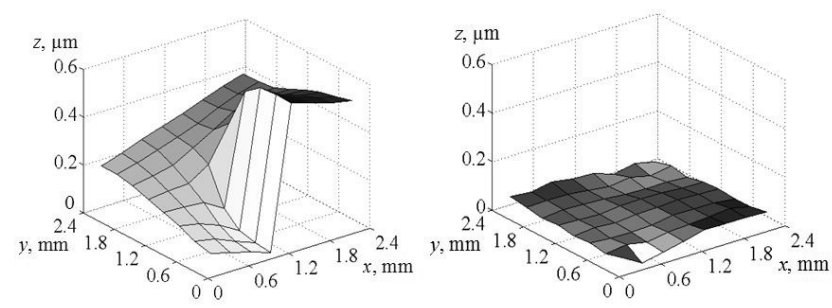

Figure 18. Experimental vortex phase surface reconstructed using modified Fried's (left) and conventional leastsquares (right) procedure.

In Figure 19 we show the calculation results [165] of phase front reconstruction of the beam passed through the turbulent atmosphere in the case of $C_{n}{ }^{2}=10^{-14} \mathrm{~cm}^{-2 / 3}$ after $1 \mathrm{~km}$ distance propagation (see Figure 4). The modified Fried's algorithm embedded into the HartmannShack sensor software correctly restores the complicated singular structure of the phase surface.

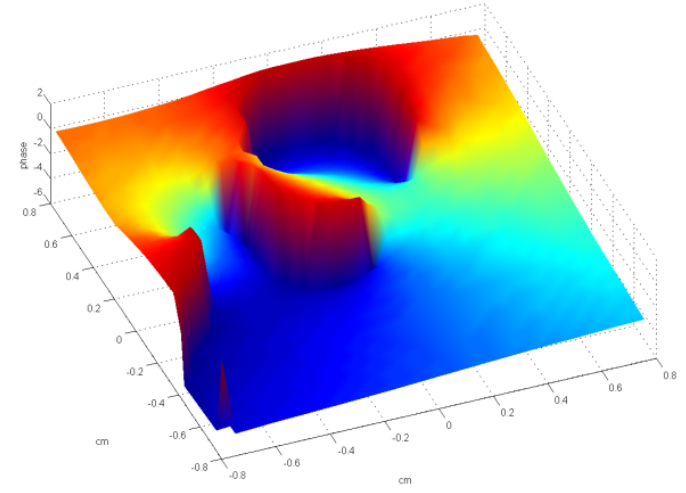

Figure 19. The phase surface fragment of the beam after the turbulent path reconstructed using the modified Fried's algorithm. 


\section{Phase correction of optical vortex}

Next we consider the possibility to transform the wavefronts of the vortex beam by means of the closed-loop adaptive optical system with a wavefront sensor and a flexible deformable wavefront corrector. We can use the bimorph [166] as well as pusher-type $[167,168]$ piezoceramic-based adaptive mirrors as a wavefront corrector. In the experiments a flexible bimorph mirror [166] and the Hartmann-Shack wavefront sensor with a new reconstruction algorithm [157-159] are employed. An attempt is made to correct the laser beam carrying the optical vortex (namely, the Laguerre-Gaussian $L G_{0}{ }^{1}$ mode), i.e., to remove its singularity. The dynamic effects are not considered, the goal is the estimation of the ability of the bimorph mirror to govern the spatial features of the optical vortex. It is very interesting to determine whether the phase correction leads to full elimination of the singularity [165].

A closed-loop adaptive system intended for performance of the necessary correction of vortex wavefront is shown in Figure 20 [169]. A reference laser beam is formed using a He-Ne laser 1 , a collimator 2, and a square pinhole 3 , which restricts the beam aperture to a size of $10 \times 10 \mathrm{~mm}^{2}$. Next the laser beam passes through a 32-level spiral phase plate 5 of a diameter of $2 \mathrm{~cm}$, a fourfold telescope 6 and comes to an adaptive deformable mirror 7 . It should be noted that the laser beam with the plane phase front that passes through the spiral phase plate maximally resembles the Laguerre-Gaussian $L G_{0}{ }^{1}$ mode in the far field. In Figure 20 the wavefront corrector is situated in near rather than far field but at a relatively large distance from the spiral phase plate so that the proper vortex structure of the phase distribution is already formed in the wavefront corrector plane.

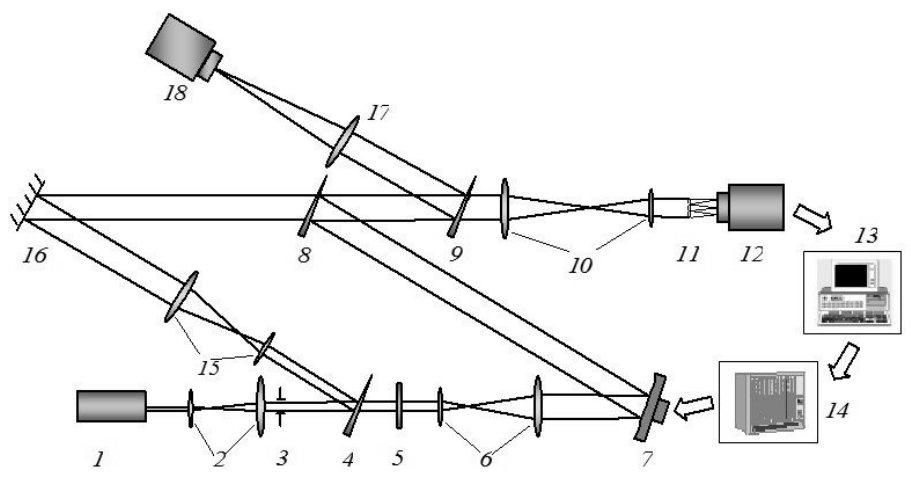

Figure 20. The close-loop adaptive system for optical vortex correction:1 - He-Ne laser; 2 - collimator; 3 - pinhole $10 \times 10 \mathrm{~mm} ; 4,8,9$ - optical plates; 5 -spiral phase plate; 6, 10, 15 - telescopes; 7 - deformable adaptive mirror; 11 lenslet array; 12, 18-CCD cameras; 13 -computer; 14 - control unit of adaptive mirror, 16 - plane mirror; 17 -lens.

The wavefront corrector (the bimorph adaptive mirror) 7 [166] is shown in Figure 21. It is composed of a substrate of LK-105 glass with reflecting coating and two foursquare piezoceramic plates, each measuring $45 \times 45 \mathrm{~mm}$ and $0.4 \mathrm{~mm}$ thick. The first piezoplate is rigidly glued to rear side of the substrate. It is complete, meaning it serves as one electrode, and is 
intended to compensate for the beam defocusing if need be. The second piezoplate destined to transform the vortex phase surface is glued to the first one. The $5 \times 5=25$ electrodes are patterned on the surface of the second piezoplate in the check geometry (close square packing). Each electrode has the shape of a square, with each side measuring $8.5 \mathrm{~mm}$. The full thickness of the adaptive mirror is $4.5 \mathrm{~mm}$. The wavefront corrector is fixed in a metal mounting with a square $45 \times 45 \mathrm{~mm}$ window. The surface deformation of the adaptive mirror under the maximal voltage $\pm 300 \mathrm{~V}$ applied to any one electrode reaches $\pm 1.5 \mu \mathrm{m}$.

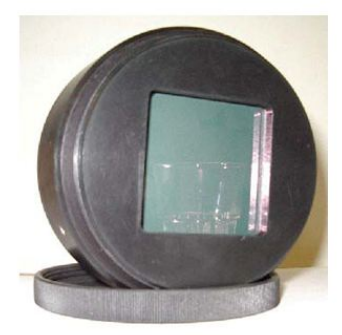

\begin{tabular}{|l|l|l|l|l|}
\hline 25 & 26 & 11 & 12 & 13 \\
\hline 24 & 10 & 3 & 4 & 14 \\
\hline 23 & 9 & 2 & 5 & 15 \\
\hline 22 & 8 & 7 & 6 & 16 \\
\hline 21 & 20 & 19 & 18 & 17 \\
\hline
\end{tabular}

Figure 21. The deformable bimorph mirror and the scheme of arrangement of control elements on the second piezoplate.

The radiation beam reflected from the adaptive mirror 7 (see Figure 20) is directed by a plane mirror 8 through a reducing telescope 10 to a Hartmann-Shack sensor including a lenslet array 11 with $d=0.2 \mathrm{~mm}, f=15 \mathrm{~mm}$ and a CCD camera 12. At the field size of $3.2 \mathrm{~mm}$ there are 16x16 spots in the hartmannogram on the CCD camera screen. The planes of adaptive mirror and lenslet array are optically conjugated so that the wavefront sensor reconstructs in fact the phase surface of the beam just in the corrector plane.

A beam part is derived by a dividing plate 9 to a CCD camera 18 for additional characterization (see Figure 20). In addition, the wavefront corrector 7, plates 4, 8 and rear mirror 16 form a Mach-Zehnder interferometer. On blocking the reference beam from the mirror 16, the CCD cameras 12 and 18 simultaneously register, respectively, the hartmannogram and intensity picture of the beam going from the adaptive mirror. Upon admission of the reference beam from the mirror 16, the CCD camera 18 registers the interference pattern of the beam going from the adaptive mirror with an obliquely incident reference beam. Screen of CCD camera 18 is situated at a focal distance from the lens 17 or in a plane of the adaptive mirror image (like the lenslet array) thus registering the intensity/interferogram of the beam in far or near field, respectively.

The wavefront has no singularity upon removal of the spiral phase plate 5 from the scheme in the Figure 20 and when switching off the wavefront corrector. The reference beam phase surface in the corrector plane is shown in Figure 22a. It is not an ideal plane ( $P V=0.33 \mu)$ but it is certainly regular. Therefore the picture of diffraction at the square diaphragm 3 (see Figure 20) roughly takes place in far field in Figure 23a. 

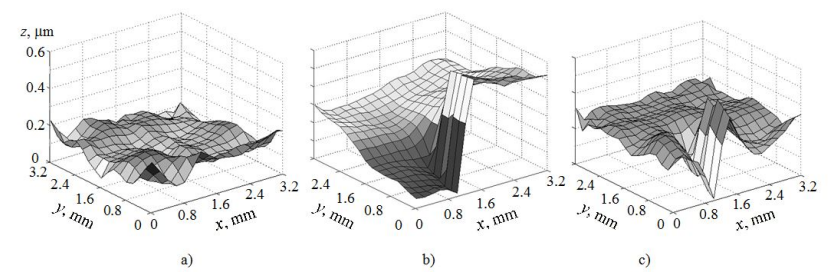

Figure 22. Experimental phase surface in near field: (a) reference beam and beam (b) before and (c) after correction.

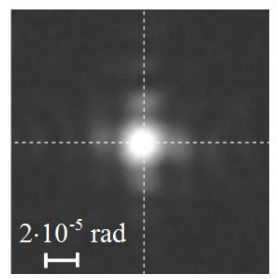

a)

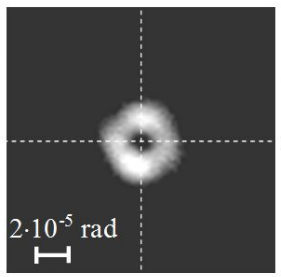

b)

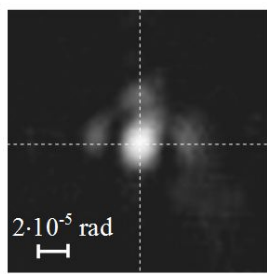

c)

Figure 23. Experimental far field intensity: (a) reference beam and beam (b) before and (c) after correction.

After inserting the spiral phase plate 5 and when switching off the adaptive mirror, the wavefront in near field in Figure $22 \mathrm{~b}$ acquires the spiral form with $\lambda$-break ( $P V=0.63 \mu$ ) so the far-field intensity has a doughnut form (see Figure 23b). Note that the wavefront corrector software in the computer 13 is based on the singular reconstruction technique [157-159]; the conventional least-squares approach fails here. The vortex in far field is the $L G_{0}{ }^{1}$ mode distorted by presence of other modes mainly because of the phase surface imperfection of the reference beam. Note that for the task of adjusting the vortex wavefront sensing technique it was necessary to form a close-to-ideal $L G_{0}{ }^{1}$ mode as a "reference" optical vortex with maximally predetermined phase surface, to determine the sensing algorithm accuracy. Here, for the correction task, it is even more attractive to work with a distorted vortex.

In order to correct the vortex wavefront in the closed loop, the recovered phase surface in Figure $22 \mathrm{~b}$ is decomposed on the response functions of control elements of the deformable mirror. The response function of a control element is the changing of the shape of the deformable mirror surface upon the energizing of this control element with zero voltages applied to the others actuators. The expansion coefficients on response functions are proportional to voltages to be applied from control unit 14 to appropriate elements of the deformable mirror. When applying control voltages to the adaptive mirror its surface is deformed to reproduce the measured vortex wavefront maximally and thus to obtain a wavefront close to a plane one upon reflection from the corrector. However, each superposition of the response functions of a flexible wavefront corrector is a smooth function, and the corrector is not able to exactly reproduce the phase discontinuity of a depth of $2 \pi$. The phase sur- 
face after the correction in Figure 22c is close to the reference one (Figure 22a) except for a narrow region at the break line $(\mathrm{PV}=0.5 \mu)$. As the radiation from this part of the beam is scattered to larger angles and its portion in the beam is relatively small, the far field intensity picture after the correction in Figure 23c is much closer to the reference beam (Figure 23a) rather than the vortex before correction (Figure 23b). Thus, the doughnut-like vortex beam is focused into a beam with a bright axial spot and weaker background that radically increases the Strehl ratio and resolution of the optical system.

The beam interferograms in near field before and after correction are shown in Figure 24 . Unlike the former, the latter contains no resolved singularities (at least, under the given fringe density). The vortices, however, may appear under beam propagation from the adaptive mirror plane as it was in the case of combined propagation of the vortex beam with a regular beam [170]. The experimental and calculated (at the reflection of an ideal $L G_{0}{ }^{1}$ mode from the actual deformed adaptive mirror surface) interferograms of a corrected beam in far field are shown in Figure 25. Two off-axis vortices (denoted by light circles) of opposite topological charge are seen here. The first of them is initial vortex shifted from the axis whereas the second arises in the process of beam propagation from the far periphery of the beam (in fact from infinity, according to the terminology of [170]).
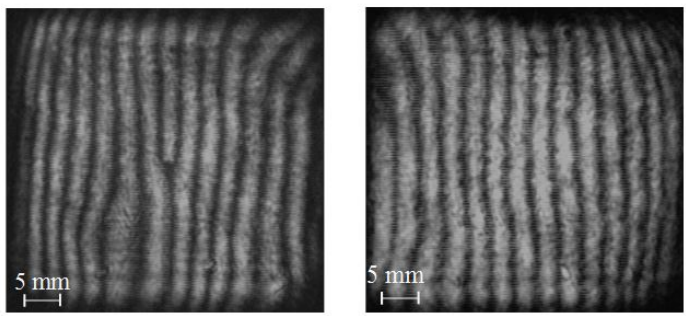

Figure 24. Experimental pattern of interference of the beam with an obliquely incident regular wave in the near field (left) before and (right) after correction.
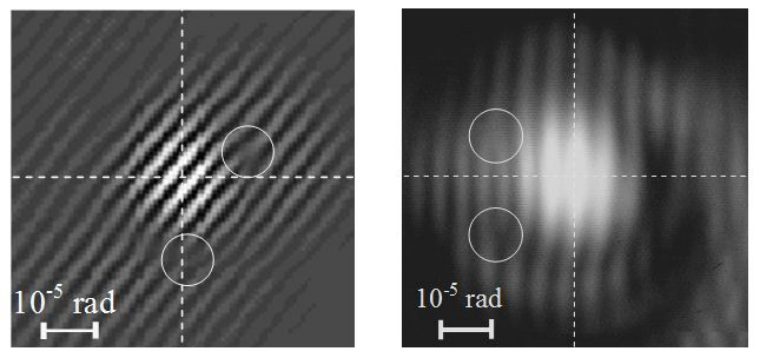

Figure 25. The pattern of interference of the corrected beam with an obliquely incident regular wave in the far field in (left) calculation and (right) experiment. 
Thus, the phase surface of the distorted $L G_{0}{ }^{1}$ mode is corrected in the closed-loop adaptive optical system, including the bimorph piezoceramic mirror and the Hartmann-Shack wavefront sensor with the singular reconstruction technique. Experiments demonstrate the ability of the bimorph mirror to correct the optical vortex in a practical sense, namely, to focus the doughnut-like beam into a beam with a bright axial spot that considerably increases the Strehl ratio and optical system resolution. Since the phase break is not reproduced exactly on the flexible corrector surface, the off-axis vortices can appear in far field at the beam periphery.

\section{Conclusions}

This chapter is dedicated to research of the possibility to control the phase front of a laser beam carrying an optical vortex by means of linear adaptive optics, namely, in the classic closed-loop adaptive system including a Hartmann-Shack wavefront sensor and a deformable mirror. On the one hand, the optical vortices appear randomly under beam propagation in the turbulent atmosphere, and the correction of singular phase front presents a considerable problem for tasks in atmospheric optics, astronomy, and optical communication. On the other hand, the controllable optical vortices have very attractive potential applications in optical data processing and many other scientific and practical fields where the regulation of singular phase is needed. This chapter discusses the main properties and applications of optical vortices, the problem of adaptive correction of singular phase in turbulent atmosphere, the issues of generating the "reference" laser vortex beam, its wavefront sensing and phase correction in the widespread adaptive optical system including a Hartmann-Shack wavefront sensor and a flexible deformable mirror.

The vortex beam is generated with help of a spiral phase plate made of fused quartz by kinoform technology. Provided that the optical quality of the spiral phase plate is good, such a means of vortex formation seems to be more preferable as compared with other considered methods of vortex generation with a well-determined phase surface. As a result, it becomes possible to obtain a singular beam very close to a Laguerre-Gaussian $L G_{0}{ }^{1}$ mode with a welldetermined singular phase structure that is necessary for checking the accuracy of subsequent wavefront reconstruction. The developed spiral phase plates are characterized by high laser damage resistance, the good surface profile accuracy and they facilitate formation of a high quality optical vortex.

The vortex phase surface measurement is carried out by a Hartmann-Shack wavefront sensor which is simpler in design and construction, more reliable and more widespread in various fields of adaptive optics when compared with other types of sensors. The commonly accepted Hartmann-Shack wavefront reconstruction is performed on the basis of the leastmean-square approach. This approach works well in the case of continuous phase distributions but is completely unsuitable for singular phase distributions. Therefore a new reconstruction technique has been developed for the reconstruction of singular phase surface, starting from the measured phase gradients. The measured shifts of focal spots in the hartmannogram are in good agreement with the calculation results. Using new software in 
the Hartmann-Shack sensor, the reconstruction of the "reference" vortex phase surface has been carried out to a high degree of accuracy.

The vortex laser beam (distorted $L G_{0}{ }^{1}$ mode) is corrected in the closed-loop adaptive system including a Hartmann-Shack wavefront sensor with singular reconstruction technique and a flexible bimorph piezoceramic mirror with $5 \times 5$ actuators allocated in the check geometry. The mirror has high laser damage resistance meaning it can operate with powerful laser beams. The purpose of the correction is to eliminate the singularity of the beam to the highest degree possible. Experiments have demonstrated the ability of the bimorph mirror to correct the optical vortex in a practical sense. As a result of phase correction, the doughnutlike beam is focused into a beam with a bright axial spot that considerably increases the Strehl ratio and is important for practical applications. However, since the wavefront break cannot be reproduced exactly by a mirror with a flexible surface, the residual off-axis vortices can appear in far field at the beam periphery.

The investigations described above consolidate the actual birth of the experimental field of novel scientific branch - singular adaptive optics.

\section{Author details}

S. G. Garanin ${ }^{1^{*}}$, F. A. Starikov ${ }^{1}$ and Yu. I. Malakhov²*

*Address all correspondence to: malakhov@istc.ru

1 Russian Federal Nuclear Center-VNIIEF, Institute of Laser Physics Research, Russia

2 International Science and Technology Center, Russia

\section{References}

[1] Vorontsov, M. A., \& Shmalgauzen, V. I. (1985). Principles of adaptive optics. Moscow: Nauka.

[2] Roggemann, M. C., \& Welsh, B. M. (1996). Imaging through turbulence. Boca Raton, FL: CRC Press.

[3] Tyson, R. K. (1998). Principles of adaptive optics. Boston, MA: Academic.

[4] Hardy, J. W. (1998). Adaptive optics for astronomical telescopes. New York: Oxford University Press.

[5] Roddier, F. (1999). Adaptive optics in astronomy. Cambridge: Cambridge University Press. 
[6] Tyson, R. K. (2000). Introduction to adaptive optics. Bellingham, WA: SPIE, International Society for Optical Engineering.

[7] Optical Vortices. (1999). Vasnetsov M, Staliunas K. (eds.) Horizons in World Physics, 228, New York: Nova Science.

[8] Soskin, M. S., \& Vasnetsov, M. V. (2001). Singular optics. Wolf E. (ed.) Progress in Optics, V.XLII. Amsterdam: Elsevier, 219-276.

[9] Bekshaev, A., Soskin, M., \& Vasnetsov, M. (2009). Paraxial light beams with angular momentum. Schulz M. (ed.) Progress in Optics Research. New York: Nova Science Publishers, 1-75.

[10] Berry, M. (1981). Singularities in waves and rays. Balian R., Kleman M., Poirier J.-P. (ed.) Physics of Defects. Amsterdam: North-Holland, 453-543.

[11] Bryngdahl, O. (1973). Radial- and circular-fringe interferograms. J. Opt. Soc. Am, 63(9), 1098-1104.

[12] Nye, J. F., \& Berry, M. V. (1974). Dislocations in wave trains. Proc. R. Soc. A, 336, 165-190.

[13] Rozas, D., Law, C. T., \& Swartzlander, G. A. Jr. (1997). Propagation dynamics of optical vortices. J. Opt. Soc. Am. B, 14(11), 3054-3065.

[14] Sacks, Z. S., Rozas, D., \& Swartzlander, G. A. Jr. (1998). Holographic formation of optical-vortex filaments. Jr. Opt. Soc. Am. B, 15(8), 2226.

[15] Coullet, P., Gil, L., \& Rocca, F. (1989). Optical vortices. Optics Commun, 73(5), 403-408.

[16] Siegman, A. E. (1986). Lasers. Sausalito, CA: University Science Books.

[17] Kogelnik, H., \& Li, T. (1966). Laser beams and resonators. Applied Optics, 5(10), 1550-1567.

[18] Allen, L., Beijersbergen, M. W., Spreeuw, R. J. C., \& Woerdman, J. P. (1992). Orbital angular momentum of light and the transformation of Laguerre-Gaussian modes. Phys Rev. A, 45(11), 8185-8189.

[19] Allen, L., Padjett, M. J., \& Babiker, M. (1999). Orbital angular momentum of light. Wolf E. (ed.) Progress in Optics, 34, Amsterdam: Elsevier, 291-370.

[20] Miller, D. A. B. (1998). Spatial channels for communicating with waves between volumes. Optics Letters, 23(21), 1645-1647.

[21] Gibson, G., Courtial, J., Padgett, M., Vasnetsov, M., Pas'ko, V., Barnett, S., \& FrankeArnold, S. (2004). Free-space information transfer using light beams carrying orbital angular momentum. Optics Express, 12(22), 5448-5456.

[22] Bouchal, Z., \& Celechovsky, R. (2004). Mixed vortex states of light as information carriers. New J. Phys, 6(1), 131-145. 
[23] Scheuer, J., \& Orenstein, M. (1999). Optical vortices crystals: spontaneous generation in nonlinear semiconductor microcavities. Science, 285(5425), 230-233.

[24] Mandel, L., \& Wolf, E. (1995). Optical Coherence and Quantum Optics. New York: Cambridge University Press.

[25] Ashkin, A. (1992). Forces of a single-beam gradient laser trap on a dielectric sphere in the ray optics regime. Biophys. J, 61(2), 569-582.

[26] Gahagan, K. T., \& Swartzlander, G. A. Jr. (1998). Trapping of low-index microparticles in an optical vortex. J. Opt. Soc. Am. B, 15(2), 524-534.

[27] Gahagan, K. T., \& Swartzlander, G. A. Jr. (1999). Simultaneous trapping of low-index and high-index microparticles observed with an optical-vortex trap. J. Opt. Soc. Am. B, 16(4), 533-539.

[28] Curtis, J. E., Koss, B. A., \& Grier, D. G. (2002). Dynamic holographic optical tweezers. Optics Commun, 207(1-6), 169-175.

[29] Ladavac, K., \& Grier, D. G. (2004). Microoptomechanical pump assembled and driven by holo- graphic optical vortex arrays. Optics Express, 12(6), 1144-1149.

[30] Daria, V., Rodrigo, P. J., \& Glueckstad, J. (2004). Dynamic array of dark optical traps. Appl. Phys. Lett, 84(3), 323-325.

[31] Khonina, S. N., Kotlyar, V. V., Shinkaryev, M. V., Soifer, V. A., \& Uspleniev, G. V. (1992). J. Mod. Optics, 39(5), 1147-1154.

[32] Swartzlander, G. A. Jr. (2001). Peering into darkness with a vortex spatial filter. Optics Letters, 26(8), 497-499.

[33] Rouan, D., Riaud, P., Boccaletti, A., Clénet, Y., \& Labeyrie, A. (2000). The four-quadrant phase-mask coronagraph. I. Principle. Pub. Astron. Soc. Pacific, 112, 1479-1486.

[34] Boccaletti, A., Riaud, P., Baudoz, P., Baudrand, J., Rouan, D., Gratadour, D., Lacombe, F., \& Lagrange-M, A. (2004). The four-quadrant phase-mask coronagraph. IV. First light at the very large telescope. Pub. Astron. Soc. Pacific, 116, 1061-1071.

[35] Foo, G., Palacios, D. M., \& Swartzlander, G. A. Jr. (2005). Optical vortex coronagraph. Optics Letters, 30(24), 3308-3310.

[36] Lee, J. H., Foo, G., Johnson, E. G., \& Swartzlander, G. A. Jr. (2006). Experimental verification of an optical vortex coronagraph. Phys. Rev. Lett, 97(5), 053901-1.

[37] Davis, J. A., Mc Namara, D. E., \& Cottrell, D. M. (2000). Image processing with the radial Hilbert transform: theory and experiments. Optics Letters, 25(2), 99-101.

[38] Larkin, K. G., Bone, D. J., \& Oldfield, M. A. (2001). Natural demodulation of two-dimensional fringe patterns. I. General background of the spiral phase quadrature transform. J. Opt. Soc. Am. A, 18(8), 1862-1870. 
[39] Masajada, J., Popiołek-Masajada, A., \& Wieliczka, D. M. (2002). The interferometric system using optical vortices as phase markers. Optics Commun, 207(1), 85-93.

[40] Senthilkumaran, P. (2003). Optical phase singularities in detection of laser beam collimation. Applied Optics, 42(31), 6314-6320.

[41] Westphal, V., \& Hell, S. W. (2005). Nanoscale Resolution in the Focal Plane of an Optical Microscope. Phys. Rev. Lett, 94(14), 143903-1.

[42] Levenson, M. D., Ebihara, T. J., Dai, G., Morikawa, Y., Hayashi, N., \& Tan, S. M. (2004). Optical vortex mask via levels. J. Microlithogr. Microfabr. Microsyst, 3(2), 293-304.

[43] Menon, R., \& Smith, H. I. (2006). Absorbance-modulation optical lithography. J. Opt. Soc. Am. A, 23(9), 2290-2294.

[44] Mair, A., Vaziri, A., Weihs, G., \& Zeilinger, A. (2001). Entanglement of the orbital angular momentum states of photons. Nature, 412(7), 313-316.

[45] Arnaut, H. H., \& Barbosa, G. A. (2000). Orbital and intrinsic angular momentum of single photons and entangled pairs of photons generated by parametric down-conversion. Phys. Rev. Lett, 85(2), 286-289.

[46] Franke-Arnold, S., Barnett, S. M., Padgett, M. J., \& Allen, L. (2002). Two-photon entanglement of orbital angular momentum states. Phys. Rev. A, 65(3), 033823.

[47] Abo-Shaeer, J. R., Raman, C., Vogels, J. M., \& Ketterle, W. (2001). Observation of vortex lattices in Bose-Einstein condensates. Science, 292(5516), 476-479.

[48] Dholakia, K., Simpson, N. B., Padgett, M. J., \& Allen, L. (1996). Second-harmonic generation and the orbital angular momentum of light. Phys. Rev. A, 54(5), R3742-3745.

[49] Starikov, F. A., \& Kochemasov, G. G. (2001). Novel phenomena at stimulated Brillouin scattering of vortex laser beams. Optics Commun, 193(1-6), 207-215.

[50] Starikov, F. A., \& Kochemasov, G. G. (2001). Investigation of stimulated Brillouin scattering of vortex laser beams. Proc. SPIE, 4403-217.

[51] Starikov, F. A. (2007). Stimulated Brillouin scattering of Laguerre-Gaussian laser modes: new phenomena. Gaponov-Grekhov AV., Nekorkin VI. (ed). Nonlinear waves 2006. N.Novgorod: IAP RAS, 206-221.

[52] Starikov, F. A., Dolgopolov, Yu. V., Kopalkin, A. V., et al. (2006). About the correction of laser beams with phase front vortex. J. Phys. IV, 133, 683-685.

[53] Starikov, F. A., Dolgopolov, Yu. V., Kopalkin, A. V., et al. (2008). New phenomena at stimulated Brillouin scattering of Laguerre-Gaussian laser modes: theory, calculation, and experiments. Proc. SPIE, 70090E, 1-11.

[54] Kopalkin, A. V., Bogachev, V. A., Dolgopolov, Yu. V., et al. (2011). Conjugation and transformation of the wave front by stimulated Brillouin scattering of vortex Laguerre-Gaussian laser modes. Quantum Electronics, 41(11), 1023-1026. 
[55] Baranova, N. B., Zel'dovich, B., Ya., Mamaev. A. V., Pilipetskii, N. V., \& Shkunov, V. V. (1981). Dislocations of the wavefront of a speckle-inhomogeneous field (theory and experiment). Sov. Phys. JETP Lett, 33(4), 195-199.

[56] Baranova, N. B., Zel'dovich, B. Ya., Mamaev, A. V., Pilipetskii, N. V., \& Shkunov, V. V. (1982). Dislocation density on wavefront of a speckle-structure light field. Sov. Phys. JETP, 56(5), 983-988.

[57] Baranova, N. B., Mamaev, A. V., Pilipetskii, N. V., Shkunov, V. V., \& Zel'dovich, B. Ya. (1983). Wavefront dislocations: topological limitations for adaptive systems with phase conjugation. J. Opt. Soc. Am. A, 73(5), 525-528.

[58] Ladagin, V. K. (1985). About the numerical integration of a quasi-optical equation. Questions of Atomic Science and Technology. Ser. Methods and codes of numerical solution of tasks of mathematical physics [1], 19-26.

[59] Feit, M. D., \& Fleck, J. A. Jr. (1988). Beam nonparaxiality, filament formation, and beam breakup in the self-focusing of optical beams. J. Opt. Soc. Am. B, 7(3), 633-640.

[60] Kandidov, V. P. (1996). Monte Carlo method in nonlinear statistical optics. Physics Usp, 39(12), 1243-1272.

[61] Goodman, J. W. (2000). Statistical optics. New York: Wiley.

[62] Fried, D. L., \& Vaughn, J. L. (1992). Branch cuts in the phase function. Applied Optics, 31(15), 2865-2882.

[63] Primmerman, A., Pries, R., Humphreys, R. A., Zollars, B. G., Barclay, H. T., \& Herrmann, J. (1995). Atmospheric-compensation experiments in strong-scintillation conditions. Applied Optics, 34(12), 081-088.

[64] Barchers, J. D., Fried, D. L., \& Link, D. J. (2002). Evaluation of the performance of Hartmann sensors in strong scintillation. Applied Optics, 41(6), 1012-1021.

[65] Kanev, F. Yu., Lukin, V. P., \& Makenova, N. A. (2002). Analysis of adaptive correction efficiency with account of limitations induced by Shack-Hartmann sensor. Proc. SPIE, 5026, 190-197.

[66] Ricklin, J. C., \& Davidson, F. M. (1998). Atmospheric turbulence effects on a partially coherent Gaussian beam: implication for free-space laser communication. Applied Optics, 37(21), 4553-4561.

[67] Levine, M., Martinsen, E. A., Wirth, A., Jankevich, A., Toledo-Quinones, M., Landers, F., \& Bruno, Th. L. (1998). Horizontal line-of-sight turbulence over near-ground paths and implication for adaptive optics corrections in laser communications. Applied Optics, 37(21), 4553-4561.

[68] Fried, D. L. (1998). Branch point problem in adaptive optics. J. Opt. Soc. Am. A, 15(10), 2759-2768. 
[69] Le Bigot, E. O., Wild, W. J., \& Kibblewhite, E. J. (1998). Reconstructions of discontinuous light phase functions. Optics Letters, 23(1), 10-12.

[70] Takijo, H., \& Takahashi, T. (1988). Least-squares phase estimation from the phase difference. J. Opt. Soc. Am. A, 5(3), 416-425.

[71] Aksenov, V. P., Banakh, V. A., \& Tikhomirova, O. V. (1998). Potential and vortex features of optical speckle field and visualization of wave-front singularities. Applied Optics, 37(21), 4536-4540.

[72] Arrasmith, W. W. (1999). Branch-point-tolerant least-squares phase reconstructor. J. Opt. Soc. Am. A, 16(7), 1864-1872.

[73] Tyler, G. A. (2000). Reconstruction and assessment of the least-squares and slope discrepancy components of the phase. J. Opt. Soc. Am. A, 17(10), 1828-1839.

[74] Fried, D. L. (2001). Adaptive optics wave function reconstruction and phase unwrapping when branch points are present. Optics Commun, 200(1), 43-72.

[75] Aksenov, V. P., \& Tikhomirova, O. V. (2002). Theory of singular-phase reconstruction for an optical speckle field in the turbulent atmosphere. J. Opt. Soc. Am. A, 19(2), 345-355.

[76] Rockstuhl, C., Ivanovskyy, A. A., Soskin, M. S., et al. (2004). High-resolution measurement of phase singularities produced by computer-generated holograms. Optics Commun, 242(1-3), 163-169.

[77] Baker, K. L., Stappaerts, E. A., Gavel, D., et al. (2004). High-speed horizontal-path atmospheric turbulence correction with a large-actuator-number microelectromechanical system spatial light modulator in an interferometric phase-conjugation engine. Optics Letters, 29(15), 1781-1783.

[78] Notaras, J., \& Paterson, C. (2007). Demonstration of closed-loop adaptive optics with a point-diffraction interferometer in strong scintillation with optical vortices. Optics Express, 15(21), 13745-13756.

[79] Hartmann, J. (1904). Objetivuntersuchungen. Z. Instrum [1, 1], 33-97.

[80] Shack, R. B., \& Platt, B. C. (1971). Production and use of a lenticular Hartmann screen. J. Opt. Soc. Am, 6(5), 656-662.

[81] Hardy, J. W., Lefebvre, J. E., \& Koliopoulos, C. L. (1977). Real-time atmospheric compensation. J. Opt. Soc. Am, 67(3), 360-369.

[82] Sandler, D. G., Cuellar, L., Lefebvre, M., et al. (1994). Shearing interferometry for laser-guide-star atmospheric correction at large D/ $\mathrm{r}_{0}$. J. Opt. Soc. Am. A, 11(2), 858-873.

[83] Roddier, F. (1988). Curvature sensing and compensation: a new concept in adaptive optics. Applied Optics, 27(7), 1223-1225.

[84] Rousset, G. (1999). Wave-front sensors. Roddier F. (ed.) Adaptive optics in astronomyCambridge: Cambridge University Press, 91-130. 
[85] Dorn, R. J. (2001). A CCD based curvature wavefront sensor for adaptive optics in astronomy. Dissertation for the degree of Doctor of Natural Sciences. University of Heidelberg, Germany.

[86] Ragazzoni, R. (1996). Pupil plane wavefront sensing with an oscillating prism. Journal of Modern Optics, 43(2), 289-293.

[87] Ragazzoni, R., Ghedina, A., Baruffolo, A., Marchetti, E., et al. (2000). Testing the pyramid wavefront sensor on the sky. Proc. SPIE, 4007, 423-429.

[88] Vorontsov, M., Riker, J., Carhart, G., Rao Gudimetla, V. S., Beresnev, L., Weyrauch, T., \& Roberts, L. C. Jr. (2009). Deep turbulence effects compensation experiments with a cascaded adaptive optics system using a $3.63 \mathrm{~m}$ telescope. Applied Optics, 48(1), A47-57.

[89] Bazhenov, V. Yu., Vasnetsov, M. V., \& Soskin, M. S. (1990). Laser beams with screw wavefront dislocations. Sov. Phys. JETP Lett, 52(8), 429-431.

[90] Brambilla, M., Battipede, F., Lugiato, L. A., Penna, V., Prati, F., Tamm, C., \& Weiss, C. O. (1991). Transverse Laser Patterns. I. Phase singularity crystals. Phys. Rev. A, 43(9), 5090-5113.

[91] Abramochkin, E., \& Volostnikov, V. (1991). Beam transformations and nontransformed beams. Optics Commun, 83(1, 2), 123-135.

[92] Grin', L. E., Korolenko, P. V., \& Fedotov, N. N. (1992). About the generation of laser beams with screw wavefront structure. Optics and Spectroscopy, 73(5), 1007-1010.

[93] Bazhenov, V. Y.u, Soskin, M. S., \& Vasnetsov, M. V. (1992). Screw dislocations in light wavefronts. J. Mod. Optics, 39(5), 985-990.

[94] Beijersbergen, M. W., Allen, L., van der Veen, H. E. L. O., \& Woerdman, J. P. (1993). Astigmatic laser mode converters and transfer of orbital angular momentum. Optics Commun, 96(1-3), 123-132.

[95] Beijersbergen, M. W., Coerwinkel, R. P. C., Kristensen, M., \& Woerdman, J. P. (1994). Helical- wavefront laser beams produced with a spiral phase plate. Optics Commun, 112(5-6), 321-327.

[96] Dholakia, K., Simpson, N. B., Padgett, M. J., \& Allen, L. (1996). Second harmonic generation and the orbital angular momentum of light. Phys. Rev. A, 54(5), R3742-R3745.

[97] Oron, R., Danziger, Y., Davidson, N., Friesem, A., \& Hasman, E. (1999). Laser mode discrimination with intra-cavity spiral phase elements. Optics Commun, 169(1-6), 115.

[98] Wada, A., Miyamoto, Y., Ohtani, T., Nishihara, N., \& Takeda, M. (2001). Effects of astigmatic aberration in holographic generation of Laguerre-Gaussian beam. Proc. SPIE, 4416, 376-379. 
[99] Miyamoto, Y., Masuda, M., Wada, A., \& Takeda, M. (2001). Electron-beam lithography fabrication of phase holograms to generate Laguerre-Gaussian beams. Proc. SPIE, 3740, 232-235.

[100] Zhang, D. W., \& Yuan X., -C. (2002). Optical doughnut for optical tweezers. Optics Letters, 27(15), 1351-1353.

[101] Malyutin, A. A. (2004). On a method for obtaining laser beams with a phase singularity. Quantum Electronics, 34(3), 255-260.

[102] Izdebskaya, Y., Shvedov, V., \& Volyar, A. (2005). Generation of higher-order optical vortices by a dielectric wedge. Optics Letters, 30(18), 2472-2474.

[103] Vyas, S., \& Senthilkumaran, P. (2007). Interferometric optical vortex array generator. Applied Optics, 46(15), 2893-2898.

[104] Kotlyar, V. V., \& Kovalev, A. A. (2008). Fraunhofer diffraction of the plane wave by a multilevel (quantized) spiral phase plate. Optics Letters, 33(2), 189-191.

[105] Arecchi, F. T., Boccaletti, S., Giacomelli, G., Puccioni, G. P., Ramazza, P. L., \& Residori, S. (1992). Patterns, space-time chaos and topological defects in nonlinear optics. Physica D: Nonlinear Phenomena, 61(1-4), 25-39.

[106] Indebetouw, G., \& Korwan, D. R. (1994). Model of vortices nucleation in a photorefractive phase-conjugate resonator. J. Mod. Optics, 41(5), 941-950.

[107] Soskin, M. S., \& Vasnetsov, M. V. (1998). Nonlinear singular optics. Pure and Applied Optics, 7(2), 301-311.

[108] Berzanskis, A., Matijosius, A., Piskarskas, A., Smilgevicius, V., \& Stabinis, A. (1997). Conversion of topological charge of optical vortices in a parametric frequency converter. Optics Commun, 140(4-6), 273-276.

[109] Yin, J., Zhu, Y., Wang, W., Wang, Y., \& Jhe, W. (1998). Optical potential for atom guidance in a dark hollow beam. J. Opt. Soc. Am. B, 15(1), 25-33.

[110] Darsht, B., Ya., Zel'dovich. B., Ya., Kataevskaya. I. V., \& Kundikova, N. D. (1995). Formation of a single wavefront dislocation. Zh. Eksp. Teor. Fiz, 107(5), 1464-1472.

[111] Fadeeva, T. A., Reshetnikoff, S. A., \& Volyar, A. V. (1998). Guided optical vortices and their angular momentum in low-mode fibers. Proc. SPIE, 3487, 59-70.

[112] Sobolev, A., Cherezova, T., Samarkin, V., \& Kydryashov, A. (2007). Bimorph flexible mirror for vortex beam formation. Proc. SPIE, 63462A, 1-8.

[113] Tyson, R. K., Scipioni, M., \& Viegas, J. (2008). Generation of an optical vortex with a segmented deformable mirror. Applied Optics, 47(33), 6300-6306.

[114] Vaughan, J. M., \& Willets, D. V. (1979). Interference properties of a light beam having a helical wave surface. Optics Commun, 30(3), 263-270. 
[115] Rozanov, N. N. (1993). About the formation of radiation with wavefront dislocations. Optics and Spectroscopy, 75(4), 861-867.

[116] Oron, R., Davidson, N., Friesem, A. A., \& Hasman, E. (2000). Efficient formation of pure helical laser beams. Optics Commun, 182(1-3), 205-208.

[117] Abramochkin, E., Losevsky, N., \& Volostnikov, V. (1997). Generation of spiral-type laser beams. Optics Commun, 141(1-2), 59-64.

[118] Tamm, C., \& Weiss, C. O. (1990). Bistability and optical switching of spatial patterns in laser. J. Opt. Soc. Am. B, 7(6), 1034-1038.

[119] Padgett, M., Arlt, J., Simpson, N., \& Allen, L. (1996). An experiment to observe the intensity and phase structure of Laguerre-Gaussian laser modes. Am. J. Phys, 64(1), 77-82.

[120] Petrov, D. V., Canal, F., \& Torner, L. (1997). A simple method to generate optical beams with a screw phase dislocation. Optics Commun, 143(4-6), 265.

[121] Snadden, M. J., Bell, A. S., Clarke, R. B. M., Riis, E., \& Mc Intyre, D. H. (1997). Doughnut mode magneto-optical trap. J. Opt. Soc. Am. B, 14(3), 544-552.

[122] Yoshikawa, Y., \& Sasada, H. (2002). Versatile of optical vortices based on paraxial mode expansion. J. Opt. Soc. Am. A, 19(10), 2127-2133.

[123] Courtial, J., \& Padjett, M. J. (1999). Performance of a cylindrical lens mode converter for producing Laguerre-Gaussian laser modes. Optics Commun, 159(1-3), 13-18.

[124] Bagdasarov, V. Kh., Garnov, S. V., Denisov, N. N., Malyutin, A. A., Dolgopolov, Yu. V., Kopalkin, A. V., \& Starikov, F. A. (2009). Laser system emitting 100 mJ in Laguerre-Gaussian modes. Quantum Electronics, 39(9), 785-788.

[125] Malyutin, A. A. (2006). Tunable astigmatic $\pi / 2$ converter of laser modes with a fixed distance between input and output planes. Quantum Electronics, 36(1), 76-78.

[126] Gabor, D. (1948). A new microscopic principle. Nature, 161(4098), 777-778.

[127] Leith, E., \& Upatnieks, J. (1961). New technique in wavefront reconstruction. J. Opt. Soc. Am, 51(11), 1469-1473.

[128] Heckenberg, N. R., Mc Duff, R., Smith, CP, \& White, A. G. (1992). Generation of optical phase singularities by computer-generated holograms. Optics Letters, 17(3), 221-223.

[129] Starikov, F. A., Atuchin, V. V., Dolgopolov, Yu. V., et al. (2004). Generation of optical vortex for an adaptive optical system for phase correction of laser beams with wave front dislocations. Proc. SPIE, 5572, 400-408.

[130] Starikov, F. A., Atuchin, V. V., Dolgopolov, Yu. V., et al. (2005). Development of an adaptive optical system for phase correction of laser beams with wave front dislocations: generation of an optical vortex. Proc. SPIE, 5777, 784-787. 
[131] Starikov, F. A., \& Kochemasov, G. G. (2005). ISTC Projects from RFNC-VNIIEF devoted to improving laser beam quality. Springer Proceedings in Physics, 102, 291-301.

[132] Sacks, Z. S., Rozas, D., \& Swartzlander, G. A. Jr. (1998). Holographic formation of optical-vortex filaments. J. Opt. Soc. Am. B, 15(8), 2226-2234.

[133] Kim, G. H., Jeon, J. H., Ko, K. H., Moon, H. J., Lee, J. H., \& Chang, J. S. (1997). Optical vortices produced with a nonspiral phase plate. Applied Optics, 36(33), 8614-8621.

[134] Shvedov, V. G., Izdebskaya, Ya. V., Alekseev, A. N., \& Volyar, A. V. (2002). The formation of optical vortices in the course of light diffraction on a dielectric wedge. Technical Physics Letters, 28(3), 256-260.

[135] Oemrawsingh, S. S. R., van Houwelingen, J. A. W., Eliel, E. R., Woerdman, J. P., Verstegen, E. J. K., Kloosterboer, J. G., \& Hooft, G. W. (2004). Production and characterization of spiral phase plates for optical wavelengths. Applied Optics, 43(3), 688-694.

[136] Ganic, D., Gan, X., \& Gu, M. (2002). Generation of doughnut laser beams by use of a liquid-crystal cell with a conversion efficiency near 100\%. Optics Letters, 27(15), 1351-353.

[137] Curtis, J. E., \& Grier, D. G. (2003). Structure of Optical Vortices. Phys. Rev. Lett, 90(13), 133901.

[138] Fishman, A. I. (1999). Phase optical elements - kinoforms. Soros Educ. J [12], 76-83.

[139] Kamimura, T., Akamatsu, S., Horibe, H., et al. (2004). Enhancement of surface-damage resistance by removing subsurface damage in fused silica and its dependence on wavelength. Jap. J. Appl. Phys, 43(9), L1229-L1231.

[140] Sung, J. W., Hockel, H., Brown, J. D., \& Johnson, E. G. (2006). Development of twodimensional phase grating mask for fabrication of an analog-resist profile. Applied Optics, 45(1), 33-43.

[141] Swartzlander, G. A. Jr. (2006). Achromatic optical vortex lens. Optics Letters, 31(13), 2042-2044.

[142] Atuchin, V. V., Permyakov, S. L., Soldatenkov, I. S., \& Starikov, F. A. (2006). Kinoform generator of vortex laser beams. Proc. SPIE, 6054, 1-4.

[143] Malakhov, Yu. I. (2006). ISTC projects devoted to improving laser beam quality. Proc. SPIE, 6346, 1-8.

[144] Malakhov, Yu. I., Atuchin, V. V., Kudryashov, A. V., \& Starikov, F. A. (2009). Optical components of adaptive systems for improving laser beam quality. Proc. SPIE, 7131, $1-5$.

[145] Southwell, W. H. (1980). Wave-front estimation from wave-front slope measurements. J. Opt. Soc. Am, 70(8), 998-1006.

[146] Vorontsov, M. A., Koryabin, A. V., \& Shmalgausen, V. I. (1988). Controlled optical systems. Moscow: Nauka. 
[147] Ghiglia, D. C., \& Pritt, M. D. (1998). Two-dimensional phase unwrapping: theory, algorithms, and software. New-York: Wiley.

[148] Zou, W., \& Zhang, Z. (2000). Generalized wave-front reconstruction algorithm applied in a Shack-Hartmann test. Applied Optics, 39(2), 250-268.

[149] Aksenov, V., Banakh, V., \& Tikhomirova, O. (1998). Potential and vortex features of optical speckle fields and visualization of wave-front singularities. Applied Optics, 37(21), 4536-4540.

[150] Roggemann, M. C., \& Koivunen, A. C. (2000). Branch-point reconstruction in laser beam projection through turbulence with finite-degree-of-freedom phase-only wavefront correction. J. Opt. Soc. Am. A, 17(1), 53-62.

[151] Aksenov, V. P., \& Tikhomirova, O. V. (2002). Theory of singular-phase reconstruction for an optical speckle field in the turbulent atmosphere. J. Opt. Soc. Am. A, 19(2), 345-355.

[152] Tyler, G. A. (2000). Reconstruction and assessment of the least-squares and slope discrepancy components of the phase. J. Opt. Soc. Am. A, 17(10), 1828-1839.

[153] Le Bigot, E.-O., \& Wild, W. J. (1999). Theory of branch-point detection and its implementation. J. Opt. Soc. Am. A, 16(7), 1724-1729.

[154] Aksenov, V. P., Izmailov, I. V., Kanev, F. Yu., \& Starikov., F. A. (2005). Localization of optical vortices and reconstruction of wavefront with screw dislocations. Proc. SPIE, 5894, 1-11.

[155] Aksenov, V. P., Izmailov, I. V., \& Kanev, F. Yu. (2005). Algorithms of a singular wavefront reconstruction. Proc. SPIE, 6018, 1-11.

[156] Aksenov, V. P., Izmailov, I. V., Kanev, F. Yu., \& Starikov, F. A. (2006). Screening of singular points of vector field of phase gradient, localization of optical vortices and reconstruction of wavefront with screw dislocations. Proc. SPIE, 6162, 1-12.

[157] Aksenov, V. P., Izmailov, I. V., Kanev, F. Yu., \& Starikov, F. A. (2006). Performance of a wavefront sensor in the presence of singular point. Proc. SPIE, 634133, 1-6.

[158] Aksenov, V. P., Izmailov, I. V., Kanev, F. Yu., \& Starikov, F. A. (2007). Singular wavefront reconstruction with the tilts measured by Shack-Hartmann sensor. Proc. SPIE, 63463, 1-8.

[159] Aksenov, V. P., Izmailov, I. V., Kanev, F. Yu., \& Starikov., F. A. (2008). Algorithms for the reconstruction of the singular wavefront of laser radiation: analysis and improvement of accuracy. Quantum Electronics, 38, 673-677.

[160] Starikov, F. A., Atuchin, V. V., Kochemasov, G. G., et al. (2005). Wave front registration of an optical vortex generated with the help of spiral phase plates. Proc. SPIE, 589, 1-11. 
[161] Starikov, F. A., Aksenov, V. P., Izmailov, I. V., et al. (2007). Wave front sensing of an optical vortex. Proc. SPIE, 634, 1-8.

[162] Atuchin, V. V., Soldatenkov, I. S., Kirpichnikov, A. V., et al. (2004). Multilevel kinoform microlens arrays in fused silica for high-power laser optics. Proc. SPIE, 5481, 43-46.

[163] Leach, J., Keen, S., Padgett, M., Saunter, C., \& Love, G. D. (2006). Direct measurement of the skew angle of the Poynting vector in helically phased beam. Optics Express, 14(25), 11919-11923.

[164] Starikov, F. A., Kochemasov, G. G., Kulikov, S. M., et al. (2007). Wave front reconstruction of an optical vortex by Hartmann-Shack sensor. Optics Letters, 32(16), 2291-2293.

[165] Starikov, F. A., Aksenov, V. P., Atuchin, V. V., et al. (2009). Correction of vortex laser beams in a closed-loop adaptive system with bimorph mirror. Proc. SPIE, 7131, 1-8.

[166] Starikov, F. A., Aksenov, V. P., Atuchin, V. V., et al. (2007). Wave front sensing of an optical vortex and its correction in the close-loop adaptive system with bimorph mirror. Proc. SPIE, 6747, 1-8.

[167] Bokalo, S. Yu., Garanin, S. G., Grigorovich, S. V., et al. (2007). Deformable mirror based on piezoelectric actuators for the adaptive system of the Iskra-6 facility. Quantum Electronics, 37(8), 691-696.

[168] Garanin, S. G., Manachinsky, A. N., Starikov, F. A., \& Khokhlov, S. V. (2012). Phase correction of laser radiation with the use of adaptive optical systems at the Russian Federal Nuclear Center- Institute of Experimental Physics. Optoelectronics, Instrumentation and Data Processing, 48(2), 134-141.

[169] Starikov, F. A., Kochemasov, G. G., Koltygin, M. O., et al. (2009). Correction of vortex laser beam in a closed-loop adaptive system with bimorph mirror. Optics Letters, 34(15), 2264-2266.

[170] Soskin, M. S., Gorshkov, V. N., Vasnetsov, M. V., Malos, J. T., \& Heckenberg, N. R. (1997). Topological charge and angular momentum of light beams carrying optical vortices. Phys. Rev. A, 56(5), 4064-4075. 